\title{
J-Aggregates of Amphiphilic Cyanine Dyes: Self-Organization of Artificial Light Harvesting Complexes
}

\author{
Stefan Kirstein ${ }^{1}$ and Siegfried Daehne ${ }^{2}$ \\ ${ }^{1}$ Institute of Physics, Humboldt University of Berlin, Newtonstr. 15, 12489 Berlin, Germany \\ ${ }^{2}$ Federal Institute for Materials Research and Testing, 12489 Berlin, Germany
}

Received 1 July 2006; Revised 27 September 2006; Accepted 18 October 2006

The simultaneous chemical linkage of cyanine dye chromophores with both hydrophobic and hydrophilic substituents leads to a new type of amphiphilic molecules with the ability of spontaneous self-organization into highly ordered aggregates of various structures and morphologies. These aggregates carry the outstanding optical properties of J-aggregates, namely, efficient exciton coupling and fast exciton energy migration, which are essential for the build up of artificial light harvesting systems. The morphology of the aggregates depends sensitively on the molecular structure of the chemical substituents of the dye chromophore. Accordingly, lamellar ribbon-like structures, vesicles, tubes, and bundles of tubes are found depending on the dyes and the structure can further be altered by addition of surfactants, alcohols, or other additives. Altogether the tubular structure is the most noticeable structural motif of these types of J-aggregates. The optical spectra are characterized in general by a complex exciton spectrum which is composed of several electronic transitions. The spectrum is red-shifted as a total with respect to the monomer absorption and exhibits resonance fluorescence from the lowest energy transition. For the tubular structures, the optical spectra can be related to a structural model. Although the molecules itself are strictly achiral, a pronounced circular dichroism (CD) is observed for the tubular aggregates and explained by unequal distribution of left- and right-handed helicity of the tubes. Photoinduced electron transfer (PET) reactions from the dye aggregates to electron acceptor molecules lead to superquenching which proves the delocalization of the excitation. This property is used to synthesize metal nanoparticles on the aggregate surface by photo-induced reduction of metal ions.

Copyright (c) 2006 S. Kirstein and S. Daehne. This is an open access article distributed under the Creative Commons Attribution License, which permits unrestricted use, distribution, and reproduction in any medium, provided the original work is properly cited.

\section{INTRODUCTION}

The natural light harvesting complexes (LHCs) of plants and photosynthetic bacteria are one of the most fascinating functional molecular assemblies. Their main purpose is strong absorption of light followed by fast energy transfer to neighboring LHCs and the photochemical reaction centre where an electron transfer process leads to charge separation. The effectiveness of these processes relies on two fundamental physicochemical principles. First self-organization of dye molecules, preferentially of chlorophyll derivatives mediated by proteins, into precisely ordered structures of large spatial size to obtain extraordinarily high cross-sections for light absorption. Second, extremely fast energy migration of the absorbed light within the LHC to ensure that the excitation energy is extremely fast available at any place where it is needed for photo-induced energy or electron transfer (PET) processes [1].
The clarification of the crystal structure of the LHCs of photosynthetic bacteria [2-5] has generated extensive activities to mimic LHCs by artificial systems. One interesting and straight forward concept is the synthesis of artificial chlorophyll derivatives which may self-assemble into LHC-like aggregates $[6,7]$. Another remarkable attempt is the growth of dendrites to tree-like dendrimers by synthetic chemistry [8-10]. Large effort has been spent to intercalate dye molecules in prefabricated supramolecular systems, like micelles, membranes, or nanotubes built from surfaceactive molecules [11-13]. The principle of light harvesting and sensitization is technically used in photovoltaic applications, where the dyes are adsorbed at the nanoporous surface of a $\mathrm{TiO}_{2}$ electrode. A spectrally sensitized PET reaction from the dye to the electrode is the primary step for the charge separation and the subsequent photocurrent [14]. However, in all these systems it has been rather difficult to arrange dye molecules into closely packed and long range ordered structures which is a prerequisite for the formation of extended 


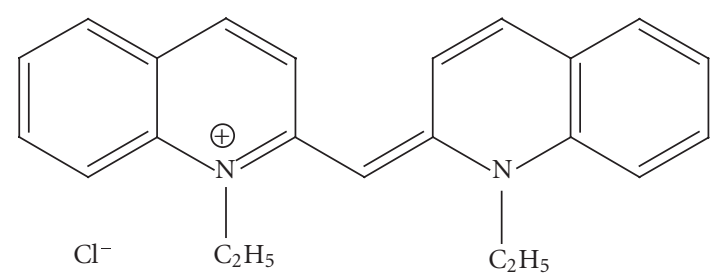

Scheme 1: Pseudoisocyanine chloride (PIC) (1).

exciton states and exciton migration across long distances as observed in natural LHCs. Most of the artificial LHCs that are studied in solution phase make use of incoherent energy transfer steps $[9,10,13]$.

In order to achieve a high degree of molecular order, it was tried to build artificial light harvesting systems by adsorbing dye molecules on solid substrates. In such heterogeneous systems, the underlying crystal lattice of the solid substrate acts as a matrix for stable fixation and precise stacking of the dye molecules by epitaxial growth $[15,16]$. Although it was studied within a completely different field of research, the first and most intensely studied PET system with coherent exciton migration was of that type, namely, sensitizing dyes and their aggregates adsorbed at silver halide crystals in photographic film emulsions [17-20]. In the early times after the sensitizing effect was observed by Vogel in 1873, only dyes were used as sensitizers which were adsorbed in their monomeric state. Here, PET happens only by chance when those dye molecules are excited that are positioned next to an active sensitivity centre of the silver halide whose energy is low enough to enable electron transfer from the dye molecule to the silver halide lattice. This was one reason for the low sensitivity of such spectrally sensitized emulsions.

The situation changed drastically after 1936, when Jelly [21] and Scheibe [22] discovered that in concentrated aqueous solutions of the spectral sensitizer pseudoisocyanine (PIC 1) (cf. Scheme 1) a new extremely small and with respect to the absorption of the monomeric molecules red-shifted absorption band appeared which was assigned to a special type of dye aggregates. Nowadays aggregates with such a behavior are named as J-aggregates or Scheibe-aggregates and the narrow absorption band is called the J-band. It was also Scheibe et al. [23] who concluded from fluorescence quenching experiments that an optical excitation within a J-aggregate should be able to migrate over thousands of molecules within a few picoseconds. Since that time the spectral sensitivity in silver halide photography has been much improved by using J-aggregates, because their energy migration ability ensured that the excitation energy is instantaneously available at any time and at any sensitivity speck of the silver halide where it is needed [18], exactly how it happens with the natural LHCs. Only 50 years later Scheibe's prediction of fast and coherent energy migration through the PIC J-aggregates, denoted in modern terms "exciton delocalization," has been experimentally confirmed by time-resolved laser spectroscopic experiments [24].

In photographic films, one condition of an effective PET by J-aggregates is their epitaxial adsorption to the silver halide lattice to induce the necessary precise ordering of the molecules. Therefore, like in other heterogeneous catalytic systems, the chemical reaction yield of the formation of metallic silver clusters (termed in photography latent image specks) is strongly limited by the size of the interfaces' surface. Although this is not important in silver halide photography, because here the silver specks are amplified through the development process by a factor up to $10^{9}$, this limitation is an important disadvantage when J-aggregates should be used as artificial LHCs in solution. The same disadvantage has to be accepted for the well-structured J-aggregates in Langmuir mono- and multilayers [25]. Therefore, homogeneous (or at least micro heterogeneous) phases are needed in order to design LHCs on the basis of J-aggregates.

In aqueous solution, most of the known spectral sensitizer dyes do not form J-aggregates or, if at all, only at rather high concentrations [17]. It is the main intention of this contribution to demonstrate a new approach to realize aggregation of homogeneous dye systems in aqueous environments with high degree of molecular order. Therefore, the self-organization ability of surfactants is combined with the coherent energy migration ability of J-aggregates. In other words, J-aggregate forming cyanine dye molecules are made amphiphilic through linkage with both strongly hydrophobic substituents and strongly hydrophilic substituents. These molecules and their respective aggregates were named as Amphipipes (amphiphiles with pigment interactions performing energy migration) $[26,27]$ in order to take into account the amphiphilic nature of the dyes and the mostly found tubelike morphology of the J-aggregates.

This paper reviews typical properties of the amphipipes with a major focus on structure and the relation between structure and optical properties. Other outstanding features of this new class of materials such as chirality or superquenching are discussed briefly. Finally, a first photoinduced electron transfer (PET) reaction between amphipipes aggregates and metal ions are presented which is considered as a promising perspective for future research. The paper essentially reports the content of the overview presented at the SOLAR'06 conference, held in Cairo.

\section{WATER SOLUBLE AMPHIPHILIC CARBOCYANINE DYES}

An outstanding feature of cyanine dyes is the very high polarizability of the $\pi$-electrons along the polymethine group in the ground state. It is at least three times as high as the polarizability of polyenes of comparable size and caused by the strong alternation of charge density. This high ground state polarizability gives rise to strong dispersion forces (van der Waals forces) between two cyanine molecules in solution $[28,29]$. Typical association enthalpies are in the order of $50-100 \mathrm{~kJ} / \mathrm{mol}$ [30-32]. For other aromatic compounds, similar high values of polarizability are only observed for the excited states which causes the formation of excimers. The dispersion forces between the ground states of polymethine chains are the main attractive forces for formation of extended aggregates of cyanines and are in general larger than 


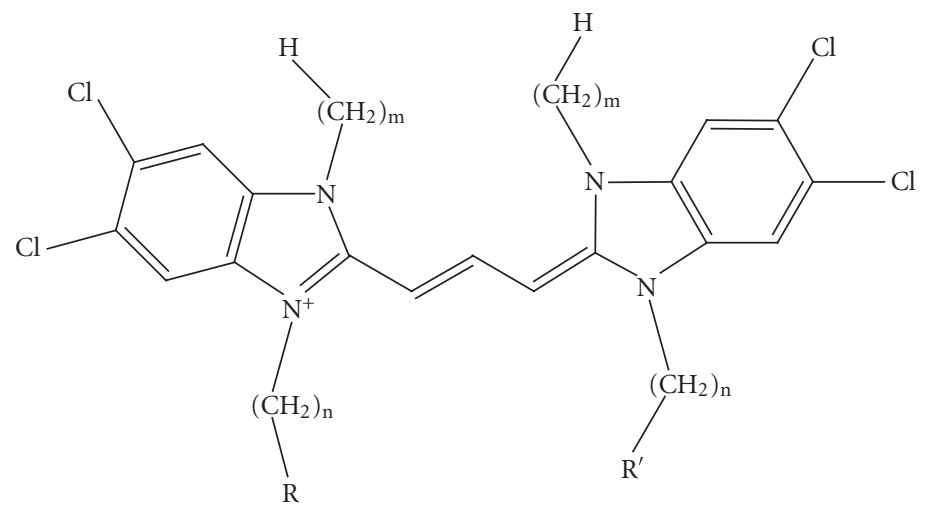

Scheme 2: 5, 5'6, 6' -tetrachlorobenzimidacarbocyanine (2). The chemical variants of (2) are identified by a mnemonic short phrase of the type $\mathbf{C} \mathbf{m R n}$, where $\mathbf{m}$ and $\mathbf{n}$ indicate the length of the alkyl chains at the $1,1^{\prime}$ - and $3,3^{\prime}-$ positions, respectively. $\mathbf{R}$ is an abbreviation to denominate the ionic groups, $\mathrm{O}$ stands for $\mathrm{R}=\mathrm{COO}^{-}$and $\mathrm{S}$ stands for $\mathrm{R}=\mathrm{SO}_{3}{ }^{-}$. See also Table 1 for further information.

the hydrophobic or van der Waals interactions between the aliphatic carbon chains or aromatic rings. As was demonstrated impressively by Daehne et al., aggregation also occurs for pure dialkylamino-polymethine chains $[33,34]$.

For most of the dyes forming J-aggregates in aqueous solutions, the morphology and structure of the aggregates are unknown. This holds even for pseudoisocyanine (PIC), the mostly investigated cyanine dye molecule, where thread-like structures polydispers in size and shape were found $[35,36]$. Only by chance, well-resolved structures such as isolated one-dimensional threads or two-dimensional leaflets [37] can be found for some other cyanine dyes. Hitherto, the only possibility to grow J-aggregates of defined structure and morphology was by using solid or liquid interfaces. In that case, the reduced dimensionality helps to arrange the molecules in macroscopically ordered structures [25].

In contrast to cyanine dyes, it is well known for surfactant molecules that they spontaneously self-assemble into regular microstructures in order to minimize unfavorable solvophobic interactions [38]. In this case, one main driving force for aggregation is the attractive hydrophobic force between alkyl chains. This force, which is caused by entropy, is in competition with the electrostatic repulsive force of the head groups and attractive van der Waals forces. The balance of all forces determines the structure of the self-assembled aggregates.

It is the main purpose of this contribution to present this new class of dye materials that link together the aggregation properties of cyanine dyes caused by dispersion forces with the self-assembly properties of surfactant molecules caused by the hydrophobic effect $[27,38-40]$. The amphiphilic character was superimposed to the dye molecules by covalently adding both hydrophobic alkyl chains and hydrophilic acid groups as substituents. In contrast to the insoluble amphiphilic dyes used for the Langmuir monolayers [25], the alkyl chains were selected shorter than dodecyl in order to provide reasonable solubility in aqueous solutions [40].

The chromophore 5, 5', 6, 6' -tetra-chloro-benz-imidacarbo-cy-anine (2) (cf. Scheme 2) has been selected from thousands of potentially J-aggregating cyanines, because it is well known that this dye with $\mathbf{m}=2, \mathrm{R}, \mathrm{R}^{\prime}=\mathrm{SO}_{3}^{-}$, and $\mathbf{n}=2$ (BIC) or $\mathbf{n}=3$ (TDBC) easily forms J-aggregates in aqueous solution even at low concentration. Additionally, these dyes are very effective spectral sensitizers in silver halide photography due to their strong negative reduction potential $(-1.61$ Volt versus saturated calomel electrode (SCE) [20]). It is well known from silver halide photography that the effectiveness of PET from photo-excited dyes into the conduction band of the silver halide strongly depends on the dyes' cathodic halfwave potential. In dependence on the dye structure, this potential may vary by more than one electron Volt even for dyes having identical light absorption, that is, identical HOMOLUMO energy gap [18-20]. Thus the J-aggregates of dyes containing chromophore 2 should be optimal suited as artificial LHCs.

Various derivatives of 2 have been synthesized following known methods $[27,40]$. Some of them are nowadays commercially available from FEW chemicals [41]. Alkyl chains of various lengths $\mathbf{m}$ were added to the nitrogen atoms at the $1,1^{\prime}$-position and different ionic groups $\mathrm{R}$ and $\mathrm{R}^{\prime}$, respectively, were attached to the nitrogen at the $3,3^{\prime}$-position via a spacer group of variable length $\mathbf{n}$. Dyes with many combinations of these three different components were synthesized and Table 1 gives a representative list of the dyes and their related aggregate structure. A short mnemonic code was introduced to address the molecules: the first two letters describe the alkyl chain of length $\mathbf{m}$ (C8 stands for octyl chains); the third letter indicates the acidic group ( $\mathrm{O}$ for carboxyl, S for sulfonic acid); and the fourth letter indicates the spacer length $\mathbf{n}$ of the ionic substituents.

The aggregation properties of these amphiphilic dyes are determined by the sensitive balance between different intermolecular forces: the dispersion force of the cyanine backbone; the entropic forces of the alkyl chains; the electrostatic forces and H-bonding of the ionic groups; and the general van der Waals forces. Due to the very high polarizability of the $\pi$-electron system of the dyes, they exhibit extraordinary high dispersion forces. For example, the free energy of aggregation for TDBC, essentially the basic chromophore of 2 , 
TABLE 1: Summary of various 5, 5', 6, 6' ${ }^{\prime}$-tetrachlorobenzimidacarbo -cyanine dyes (2) that have been synthesized and investigated. The trivial name is a mnemonic code to express the length of the alkyl and spacer chains and the chemical nature of the acidic substituents. The morphology is confirmed by electron microscopy only for the cases indicated by an asterisk $(*)$. In the other cases, it is concluded from the optical spectra.

\begin{tabular}{lll|l}
\hline \multicolumn{4}{l}{ Carboxyl compounds $(\mathrm{R}=\mathrm{COOH})$} \\
\hline $\mathrm{m}$ & $\mathrm{n}$ & Trivial name & Morphology of aggregates \\
\hline 8 & 1 & $\mathrm{C} 8 \mathrm{O} 1$ & no J-aggregates \\
\hline 8 & 2 & $\mathrm{C} 8 \mathrm{O} 2$ & tubular, chiral \\
8 & 3 & $\mathrm{C} 8 \mathrm{O} 3^{*}$ & \\
\hline 8 & 4 & $\mathrm{C} 8 \mathrm{O} 4^{*}$ & planar, achiral \\
\hline 2 & 3 & $\mathrm{C} 2 \mathrm{O} 3$ & \\
4 & 3 & $\mathrm{C} 4 \mathrm{O} 3$ & planar or linear, achiral \\
6 & 3 & $\mathrm{C} 6 \mathrm{O} 3$ & \\
\hline 7 & 3 & $\mathrm{C} 7 \mathrm{O} 3$ & \\
8 & 3 & $\mathrm{C} 8 \mathrm{O} 3$ & tubular, chiral \\
10 & 3 & $\mathrm{C} 10 \mathrm{O} 3$ & \\
11 & 3 & $\mathrm{C} 11 \mathrm{O} 3$ & \\
12 & 3 & $\mathrm{C} 12 \mathrm{O} 3$ &
\end{tabular}

Sulfo-compounds $\left(\mathrm{R}=\mathrm{SO}_{3}^{-}\right)$

\begin{tabular}{lll|l}
\hline 8 & 3 & $\mathrm{C} 8 S 3^{*}$ & tubes (single) \\
\hline 8 & 2 & $\mathrm{C} 82^{*}$ & ribbons/tubes \\
\hline 2 & 3 & BIC $(\mathrm{C} 2 \mathrm{~S} 3)$ & \\
2 & 4 & TDBC $(\mathrm{C} 2 \mathrm{~S} 4)$ & planar or linear \\
4 & 4 & $\mathrm{C} 4 \mathrm{~S} 4$ & \\
\hline
\end{tabular}

was reported to be approximately $80 \mathrm{kJmol}^{-1}$ for one dimer [42]. For the amphiphilic dyes, an additional attractive energy of the same magnitude is expected from the hydrophobic effect of the alkyl chains. Therefore, the hydrophobic substituents have a significant effect on the molecular packing. In total, the attractive interaction between the dyes is very strong which causes aggregation at rather low concentrations of typically $10^{-6} \mathrm{~mol}^{-1}$, in contrast to the pure TDBC which aggregates at $10^{-4}-10^{-3} \mathrm{~mol} \mathrm{l}^{-1}[43]$.

A crude estimate about the aggregate morphology can be made from the so-called surfactant parameter $N_{s}=V / l a_{0}$, where $V$ and $l$ represent the volume and length of the alkyl chains, respectively, and $a_{0}$ is the effective cross-sectional area per head group [38]. The value of $N_{s}$ gives an indication about the curvature of the aggregate assembly. As a rule of thumb, spherical structures (micelles) are obtained for $N_{s} \approx 0.33$, cylindrical structures (tubules) for $N_{s} \approx 0.5$, and planar bilayers (vesicles) for $N_{s} \approx 1$. For the amphipipes, a typical $N_{s}$ value in the range of 0,5 to 1 is calculated. Although the aggregate structure cannot be predicted a priori from this parameter, a cylindrical or a planar geometry seems to be most likely.

Already from the optical spectra, one can conclude that a variety of complex structures of the corresponding Jaggregates exists for different hydrophobic alkyl substituents or hydrophilic acidoalkyl substituents. After detailed study of the aggregate morphologies, as will be described in the next section, the principle structures of the aggregates can be assigned to their optical spectra. The unexpected and essential result was that there are at least two different aggregate types. One type behaves like the classical J-aggregate, showing one single narrow absorption band red-shifted likewise with respect to the monomeric absorption accompanied by resonance fluorescence from this J-band. These aggregates form mostly planar structures. The other type which is found for several of the dyes 2 exhibits a rather complex absorption spectrum that is red-shifted as a whole with respect to the monomer absorption. The typical morphology of these aggregates is a tubular structure as will be described below. The tubular aggregate structures exhibit optical activity as observed by circular dichroism (CD) spectroscopy. The observed $\mathrm{CD}$ effect indicates spontaneous and enantio-selective formation of chiral structures.

In Table 1, a list of investigated molecules $\mathbf{2}$ is compiled where the morphologies are indicated as concluded from their optical spectra. It becomes obvious that the length $\mathbf{m}$ of the alkyl chains at $1,1^{\prime}$-position as well as the spacer length $\mathbf{n}$ at the 3, $3^{\prime}$-position is mainly responsible for the shape of the aggregates.

Aggregates with optical spectra that are indicative for tubular shapes and which show optical activity are found in aqueous solutions for 3, 3'-carboxypropyl substituted dyes having alkyl groups longer than hexyl $(\mathbf{m}>6)$, like $\mathrm{C} 8 \mathrm{O} 3$ to $\mathrm{C} 12 \mathrm{O} 3$. The same behavior is observed when the carboxypropyl groups of the dioctyl derivative $\mathrm{C} 8 \mathrm{O} 3$ are replaced by carboxyethyl substituents in $\mathrm{C} 8 \mathrm{O} 2$. However, these aggregates are less stable and undergo structural transitions with time. After some days they are transformed to achiral planar J-aggregates, having one single J-absorption band.

A similar variation of the aggregate spectra with the length of the alkyl chains is observed for dyes with the much more acidic sulfoalkyl groups instead of the carboxyalkyl substituents. The $1,1^{\prime}$-diethyl substituted dyes $(\mathbf{m}=2)$ are well known in literature and usually abbreviated as BIC (C2S3, having two 3, 3'-sulfopropyl groups) and TDBC (C2S4, having two 3, $3^{\prime}$-sulfobutyl groups). Both molecules form single J-bands likewise and chirality has never been observed. But the dioctyl substituted derivatives C8S3 and C8S2 again are able to form tubular aggregates that are chiral likewise. However, the explicit form of these aggregates depends strongly on the environmental conditions [44, 45]. During the extensive investigations of the amphipipes, it came out that the dyes $\mathrm{C} 8 \mathrm{O} 3$ and $\mathrm{C} 8 \mathrm{~S} 3$ are the ideal candidates for detailed studies of optical and structural properties of tubular J-aggregates. Therefore, these aggregates will be discussed in more detail in the next sections.

Planar aggregate structures were found for dyes with $3,3^{\prime}$-carboxypropyl as well as derivatives having short alkyl chains in $1,1^{\prime}$-position (C2O3 to $\left.\mathrm{C} 6 \mathrm{O} 3\right)$. They behave like classical J-aggregates, that is, in rather concentrated aqueous solutions only one single with respect to the dye monomer red-shifted J-absorption band appears in the spectrum and the aggregates are achiral like the monomers. The 
fluorescence of these aggregates is observed from a band which is almost identical to the J-absorption band, a feature which is also typical for classical J-aggregates. A similar behavior is observed for molecules with long spacer groups, that is, $\mathbf{n}$ larger than 4 . Molecules with alkyl chains longer than octyl were not considered because of their insolubility in aqueous solution.

\section{SELECTED AGGREGATE STRUCTURES}

The aggregate structure on a molecular scale is hardly accessible by direct characterization techniques, such as x-ray crystallography. However, the morphology can be imaged on mesoscopic scales by advanced microscopy techniques such as electron microscopy and scanning force microscopy [46]. If the aggregates exhibit morphologies that are regularly shaped than it might be possible to draw conclusions about the structure on a molecular scale.

A major breakthrough in the understanding of the structure and morphology of the J-aggregates of the amphiphilic dyes was achieved by von Berlepsch et al. with the help of cryogenic transmission electron microscopy (cryo-TEM) $[37,47,48]$. Cryo-TEM is a well-established technique in biology and other areas of supramolecular chemistry to characterize water soluble structures in their native environment [49]. For this method, a thin film of the liquid sample is vitrified in liquid ethane at its freezing point $\left(-183.2^{\circ} \mathrm{C}\right)$ by shock freezing to avoid crystallization of water. The samples are transferred to the electron microscope, investigated under liquid nitrogen $\left(-175^{\circ} \mathrm{C}\right)$, and imaged in transmission mode (more details can be found in [49] and in the references of von Berlepsch et al.). In the following subsections, typical morphologies and their relation to the optical spectra are reviewed.

\subsection{Tubular aggregates}

\subsubsection{Morphology}

Tubular aggregates were first discovered by cryo-TEM for the amphiphilic molecule $\mathrm{C} 8 \mathrm{O} 3$ [37] and meanwhile they are confirmed for the dyes C8S2 [44] and C8S3 [45, 50] as well. Typical cryo-TEM images of the tubular aggregates of $\mathrm{C} 8 \mathrm{O} 3$ and C8S3 are shown in Figures 1 and 2. In the case of $\mathrm{C} 8 \mathrm{O} 3$ (Figure 1(a)), rope-like structures are visible that consist of bundles of a distinct number of tubules. For clarification, a magnified view together with a computer simulation of the cryo-TEM image is shown in Figure 1(c). The computer simulation is a drawing of hollow cylinders that are packed on a triangular lattice and twisted around each other. Using semitransparent cylinders, a projection image is created which reproduces exactly the details that are visible in the experimental cryo-TEM image.

The outer diameter of the tubules of $\mathrm{C} 8 \mathrm{O} 3$ is of the order of $10 \mathrm{~nm}$ and the length of the rope-like bundles exceeds hundreds of micrometer. From a close inspection of the cryoTEM images, the thickness of the wall of the tubules can be determined to be approximately $4 \mathrm{~nm}$. This thickness is taken as an indication that the wall consists of a bilayer of the

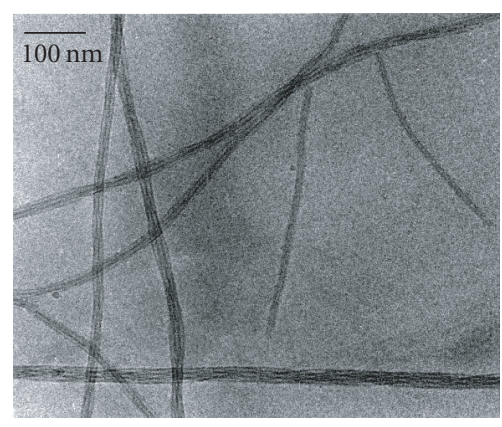

(a)

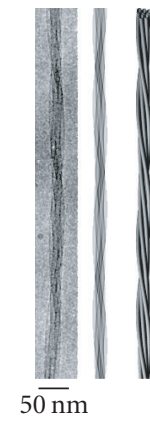

(b)

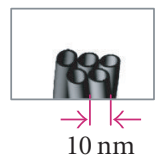

(c)
FIgure 1: Cryo-TEM image of a $2.8 \times 10^{-4} \mathrm{M}$ aqueous solution of $\mathrm{C} 8 \mathrm{O} 3$ and respective computer graphic simulation of the structure. In (a) rope-like structures are visible that are composed of tubular aggregates. A magnified view of a rope-like aggregate of $\mathrm{C} 8 \mathrm{O} 3$ together with a computer graphic image and the corresponding calculated transparent image is also shown. The computer graphic is built from tubules with a diameter of $10 \mathrm{~nm}$ that are densely packed and twisted around each other. (Adapted with permission from [37]. Copyright (2000) American Chemical Society.)

amphiphilic dye molecules similar to a lipid bilayer. In this bilayer, the chromophores and their hydrophilic side groups enclose the hydrophobic alkyl chains to protect them from the water. As a consequence of this structure model, not only the outer part, but also the inner part of the tube has hydrophilic groups and hence the whole tube should be filled with water.

The single tubules are highly monodispers in diameter, but the number of tubes forming a twisted bundle is varying in the range of 3 to more than 10 . The handedness of the twist of the bundles cannot be seen from the cryo-TEM images. For this purpose, the aggregates have to be dried on a solid substrate and imaged after oblique shadowing to resolve the three-dimensional surface. This topic will be discussed in Section 5 .

The diameter of a single tubule of $\mathrm{C} 8 \mathrm{O} 3$ is rather constant and changes only insignificantly upon addition of any additives like alcohols [51]. It even remains constant under the addition of polyalcohols or polyelectrolytes to the aggregate solution [52]. However, as will be shown below, the twisted bundle tends to separate into single tubes upon addition of alcohols. 


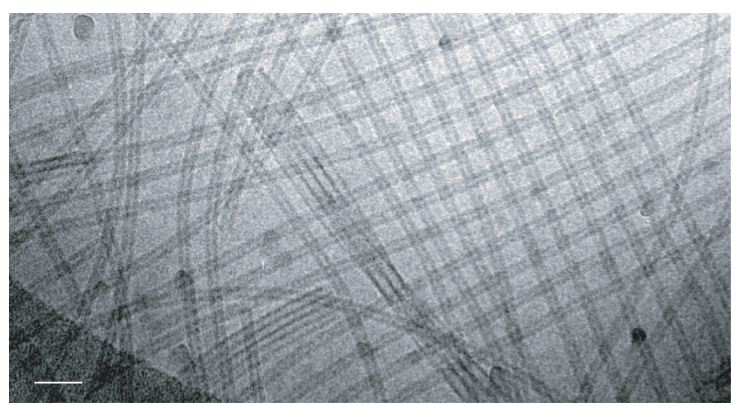

(a)

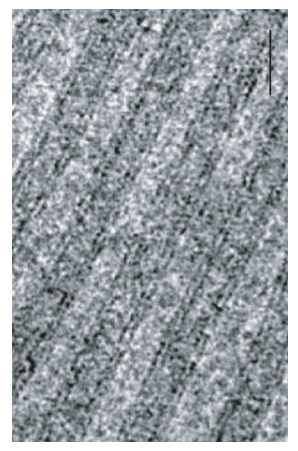

(b)

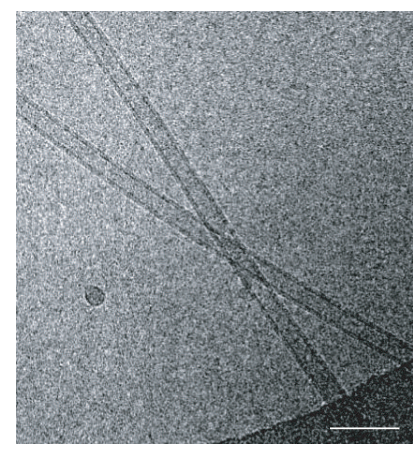

(c)
Figure 2: Cryo-TEM images of tubular J-aggregates of C8S3. (a) $5.3 \times 10^{-4} \mathrm{M}$ C8S3 sample prepared via the "alcoholic route" 3 days after preparation. $[\mathrm{MeOH}]=16 \mathrm{wt} \%$. Bar $=50 \mathrm{~nm}$. (b) Magnified view of tubular aggregates. $\mathrm{Bar}=20 \mathrm{~nm}$. (c) $3.4 \times 10^{-4} \mathrm{M} \mathrm{C} 8 \mathrm{~S} 3 \mathrm{sam}-$ ple prepared via the "direct route" 9 days after preparation. Bar $=$ $50 \mathrm{~nm}$.

Well-separated single tubules are formed by the sulfobutyl substituted dye C8S3 (see Figure 2), only sporadically bundles of tubes are found. These tubules are very monodispers in diameter and rather straight with a length of up to several micrometers. However, the diameter of these tubes depends on the preparation conditions [45]. If the molecules are dissolved in pure water the outer diameter is $16 \mathrm{~nm}$. For solutions that contain more than $18 \mathrm{vol} \%$ of $\mathrm{MeOH}$ tubes with a diameter of $13 \mathrm{~nm}$ are found. For both cases the wall thickness of the aggregates is of the order of $4 \mathrm{~nm}$. In Figure 2, both types of tubular aggregates are presented. It is interesting to note that no intermediate size of the diameter was observed. Upon addition of alcohol to a pure water solution, a conversion between these two distinct aggregate types can be generated [45].

An even stronger dependence on the solvent conditions is found for the dye C8S2. Here, tubular aggregates exist only in presence of small portions of $\mathrm{MeOH}(<4 \%)$ and at low concentration of the dye $\left(<1 \cdot 10^{-4} \mathrm{~mol} \cdot \mathrm{l}^{-1}\right)$. Under these conditions, large tubes with a diameter of $28 \mathrm{~nm}$ are observed [44].

The tubes of $\mathrm{C} 8 \mathrm{~S} 3$ are very regular structures which indicates a high molecular order. In some of the cryoTEM images, an internal superstructure becomes visible, although hidden by photographic noise. By a phase-sensitive

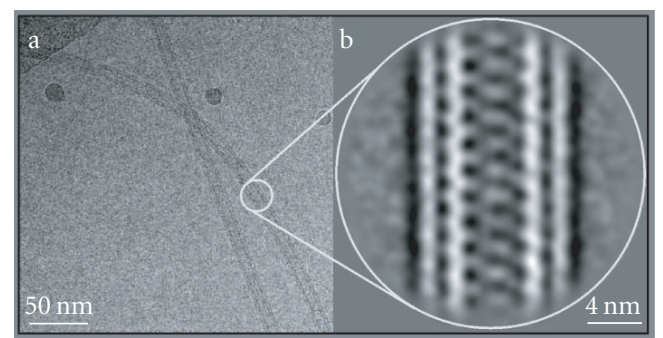

Figure 3: Magnified view of two tubular aggregates of C8S3 prepared by the direct route (cf. Figure 2(c)). By image sampling and averaging along the tubular aggregates, a high resolution transmission contrast image was evaluated, seen on the right. The doublelayer wall and the internal structure of the tubules become visible.

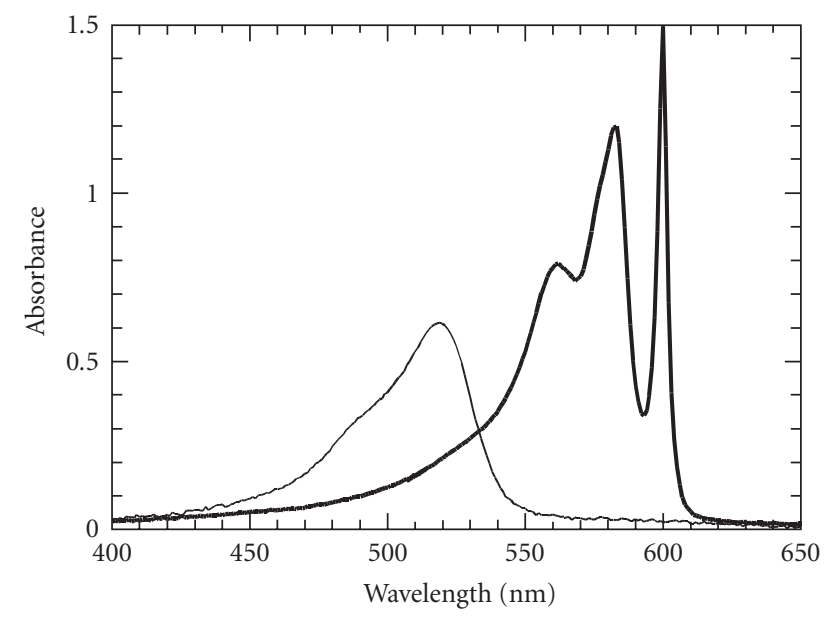

Figure 4: Absorption spectrum of a $5.0 \times 10^{-5} \mathrm{~mol} / \mathrm{l} \mathrm{C} 8 \mathrm{O} 3$ solution in $10 \mathrm{mM} \mathrm{NaOH}$. The spectrum of the monomer is drawn by a thin line for comparison. (Adapted with permission from [47]. Copyright (2000) American Chemical Society.)

superposition of fractions of images alongside one tubule the noise can be substantially reduced. The result of such an improved magnified view of part of a tubule is shown in Figure 3. The double-wall structure and an internal structure of ripples become clearly visible. The ripples are interpreted as a helix structure which here is seen in projection. This internal helix structure is directly related to the occurrence of chirality in these systems, as will be discussed below.

\subsubsection{Optical spectroscopy}

The optical absorption spectra of the tubular aggregates are characterized by the appearance of several absorption bands forming a spectrum that is in total red-shifted as compared to the monomer transition. Because of the red-shift of the whole absorption spectrum, these aggregates are named as J-aggregates as well. Typical optical absorption spectra are shown in Figures 4 and 5 for $\mathrm{C} 8 \mathrm{O} 3$ and for C8S3, respectively, together with fluorescence emission spectra for C8S3. In the case of $\mathrm{C} 8 \mathrm{O} 3$, the band with lowest transition energy 


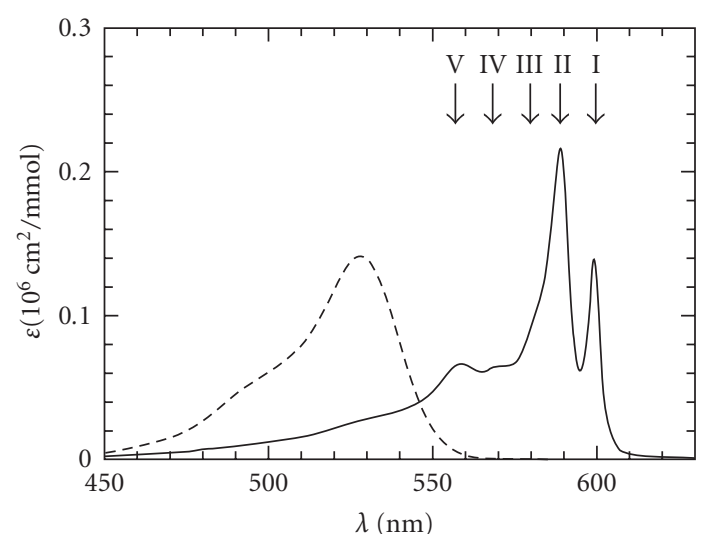

(a)

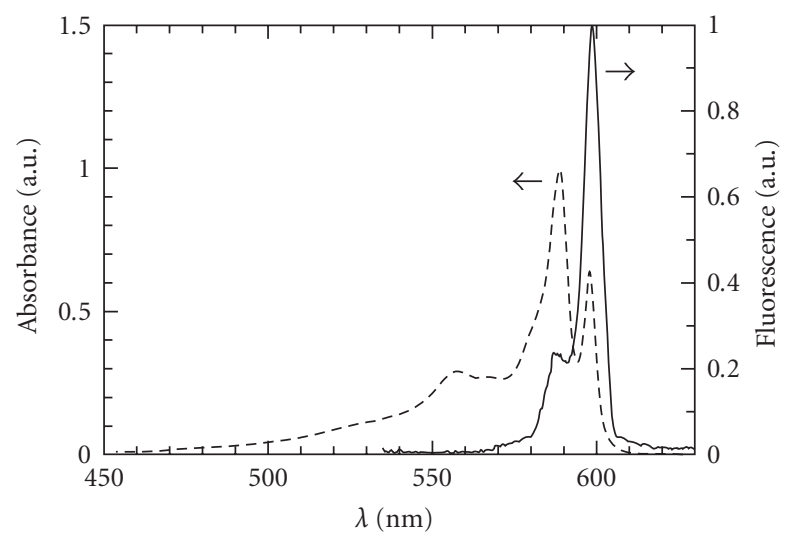

(b)

FIgURE 5: Absorption and fluorescence spectra of a C8S3 solution prepared via the "alcoholic route." (a) Absorption spectrum of $[\mathrm{C} 8 \mathrm{~S} 3]=6 \times 10^{-5} \mathrm{M}$; prepared from a $\mathrm{MeOH}$ stock solution. $[\mathrm{MeOH}]=16 \mathrm{wt} \%$. (b) Fluorescence emission spectra 1 hour after preparation via the "alcoholic route" $\left([\mathrm{C} 8 \mathrm{~S} 3]=4.33 \times 10^{-4} \mathrm{M}\right.$, $22 \mathrm{wt} \% \mathrm{MeOH})$. For the fluorescence measurement the solution was strongly diluted $\left([\mathrm{C} 8 \mathrm{~S} 3]=8.5 \times 10^{-8} \mathrm{M}\right)$.

is very narrow and exhibits fluorescence emission without Stokes shift $[39,53]$. It was proven by polarized excitation spectroscopy that all peaks of the spectrum belong to the exciton spectrum of one tubular structure $[52,54,55]$. It has to be mentioned that the characteristic features of the absorption spectra, that is, the number and position of apparent maxima, may depend on the preparation conditions. However, the spectra, and hence the structure of the aggregates, came out to be rather independent on the dye concentration. Typical concentrations for the experiments presented here are $10^{-5}$ to $10^{-3} \mathrm{~mol} / \mathrm{l}$. For the case of $\mathrm{C} 8 \mathrm{~S} 3$, the tubular aggregates could be observed by absorption and fluorescence spectroscopy even in solutions that were diluted down to $10^{-8} \mathrm{~mol} / \mathrm{l}$.

The principle structure of the spectra and their relation to the molecular aggregate structure was clarified by Pugzlys et al. measuring the polarization of the respective optical transitions $[50,52]$. For these measurements, the aggregates were oriented by shear stress in a streaming flow cell. More details about this technique and results are found in the contribution by A. Pugzlys in [56]. By measuring absorbance parallel and perpendicular to the flow direction, linear dichroism (LD) spectra were recorded for the structures of $\mathrm{C} 8 \mathrm{O} 3$ in combination with poly(vinyl alcohol) [52] and for C8S3 [45]. The spectra of both systems have essentially the same shape. The two absorption bands with lowest energy (named I and II in Figure 5(a)) are polarized along the aggregate axis, while the third peak at higher energy (III) is polarized perpendicular. The less pronounced absorption bands IV and V are not or only weakly polarized and, therefore, their origin is not completely clarified. The spectra of other tubular structures show a similar pattern, although the intensity distribution may be different. Furthermore, sometimes two or more of the absorption peaks interfere with each other which leads to spectra with only two or three visible maxima. In general, it seems to be necessary to take into account four optical transitions to describe the excitonic absorption spectrum of the tubular structures. More details are found in the contribution by J. Knoester in [57].

The fluorescence emission of the tubular structures is in resonance with the lowest energy transition, independent of the wavelength used for excitation. This behavior indicates fast energy relaxation within the exciton band into the lower energy band edge. However, recently it became evident for aggregates of C8S3 [45] and C8S2 [44] that small fraction of fluorescence also occurs from transitions at the next higher energy level. The ratio of the relative intensities of the two fluorescence peaks differs from the ratio of the corresponding absorption peaks, which indicates, that partially the energy is transferred from the higher energy state into the lower one. As an example, the fluorescence emission of $\mathrm{C} 8 \mathrm{~S} 3$ aggregates is shown in Figure 5(b).

\subsubsection{Molecular model of aggregate structure}

In our early publications, a structural model for the tubular aggregates was developed only on the basis of spectroscopic and light scattering data. The most simple model was that of a cylindrical micelle [40]. The hydrophobic alkyl chains were thought to be enclosed by the chromophores and the respective hydrophilic ionic substituents and the chromophores are following a helical screw-like stacking to account for the circular dichroism. In parallel, a similar model was used by $\mathrm{H}$. Kuhn and C. Kuhn to give a simple theoretical explanation for the optical absorption and CD spectra of the tubular aggregates [58]. From this model, the occurrence of two redshifted J-bands with perpendicular polarization and a CD doublet at the position of the absorption bands were predicted. These results were almost consistent with the spectroscopic data of $\mathrm{C} 8 \mathrm{O} 3$.

Later, the optical properties of cylindrical aggregates were studied extensively by Knoester et al. [54, 55, 59, 60] using a model where the molecules are located on rings. A cylinder surface is formed by stacking the rings on top of each other with a certain turn between two subsequent rings to simulate chirality [61]. This cylinder model was used to calculate 

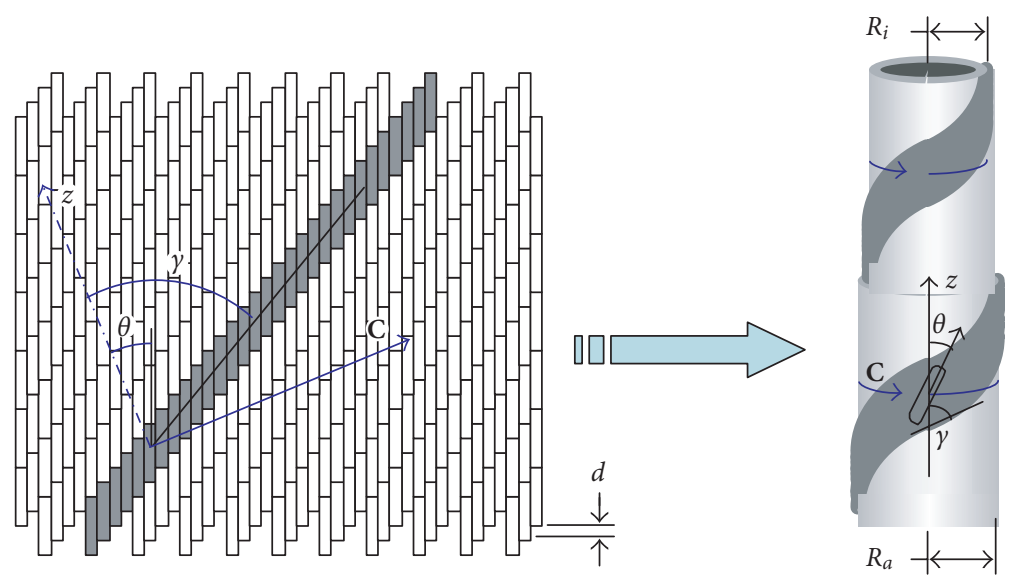

Figure 6: Structure model for calculation of exciton energies of tubular J-aggregates following the model given by Didraga et al. [50]. A two-dimensional brick work structure defines the packing of the molecules represented by rectangles. A cylinder surface is formed by rolling this lattice along the wrapping vector $\mathbf{C}$ which has to be commensurate with lattice periodicity. The $z$-direction is normal to $\mathbf{C}$, and the diameter of the cylinder is defined by the relation $2 \pi R=C$. The tilt angle $\theta$ of the molecules with respect to the $z$-axis results from the mutual shift $s$. The orientation of $\mathbf{C}$ defines the screw angle $\gamma$ of the helix that is described by a selected chain of molecules (indicated by grey shadowing). Two of those cylinder surfaces with different radii $R_{i}$ and $R_{a}$ are concentrically arranged to form the double-layer wall. In the Figure, the two cylinders are sketched in an extracted state like a telescope.

the linear and third order nonlinear absorption spectra [60]. Later, it was modified in order to include chirality and molecular disorder into the calculations [59, 61, 62]. Also this model was successfully applied to describe some linear and nonlinear optical properties of aggregates of $\mathrm{C} 8 \mathrm{O} 3$.

Based on the spectroscopic characterization of tubular aggregates of $\mathrm{C} 8 \mathrm{~S} 3$ and the more detailed structure information (cf. Figure 3), a more realistic structural model was developed to calculate exciton bands by Didraga et al. [50]. Although this model was originally applied to describe the spectra of aggregates of $\mathrm{C} 8 \mathrm{~S} 3$, it seems that the principle features of this model are transferable to all other tubular aggregate structures [51]. This double-layer model of Didraga et al. is presented in Figure 6. The starting point is a twodimensional brick-layer that represents the cyanine chromophores. The brick-layer structure is characterized by the size of the molecules and the shift $s$ between two neighboring molecules. The interaction energy of the transition dipoles is mostly determined by this shift and it is known that the typical J-aggregate behavior, namely, a low energy absorption band, is related to a large shift $s$. The magnitude of this shift is a property of the chromophore itself. It cannot be changed largely by the chemical side groups. Such a brick layer is rolled into a cylindrical surface along a wrapping vector $\mathbf{C}$ giving a circumference of $C=2 \pi R$. Because of the periodic boundary conditions, only certain choices of $\mathbf{C}$ are alloweda fact which could explain the very uniform and monodispers diameter of the tubules. In order to take into account the bilayer structure of the aggregates, two of these cylinders are concentrically stacked into each other where the radii differ by approximately $4 \mathrm{~nm}$. By the choice of $\mathbf{C}$, two important angles are fixed: the tilt angle $\theta$ of the long molecular axis with respect to the $z$-axis of the tubule and the screw-angle $\gamma$ of a line of molecules. The latter is not independent but depends implicitly on the shift $s$. The helical winding of rows of molecules is indicated in Figure 6 by grey shadowing. It is obvious from this model how helicity and hence chirality appears in the supramolecular structure although the basic elements are completely symmetric and achiral. A screw-like internal structure as indicated in Figure 6 was indeed observed by high resolution cryo-TEM for the tubular aggregates of C8S3, see [63, Figure 3].

The calculation of the linear optical properties, such as exciton energies and dipole strength based on this model is straight forward and the details are given by Knoester in [57] and can be found in [50]. The energy levels of the optical transitions were calculated using the extended dipole model developed by Czikkely et al. [64]. As in the case of a circular aggregate, for one cylinder structure, two exciton states with allowed optical transitions are obtained, both shifted towards lower energies compared to the monomer transition. The exciton level with the lower energy is polarized along the aggregate axis while the other is polarized perpendicular. The ratio of oscillator strengths is solely determined by the tilt angle $\theta$. For a fixed shift $s$ of the molecules, the energy levels are mostly dependent on the length and direction of the rolling vector $\mathbf{C}$ and therefore strongly depend on the diameter of the cylinders and tubules. Because of the two different cylinders needed to describe the bilayer in total, four excitonic transitions are obtained to describe the complete spectrum. This explains the different shape of the spectra of the various tubular structures. For different tube radii, the position of the exciton bands is slightly shifted and sometimes the bands of the transitions overlap. Therefore, for some tubular 
aggregates only two or three pronounced peaks are visible in the absorption spectrum and the remaining transitions are only noticeable as shoulders.

This model was explicitly applied to simulate the experimentally measured spectrum of the aggregates of C8S3 (cf. Figure 5). A best fit between calculated and measured energy levels could be obtained using an inner and outer diameter of 10.9 and $15.7 \mathrm{~nm}$ and tilt angles of $47.4^{\circ}$ and $43.0^{\circ}$, respectively [50].

It is interesting to note that within this model approximately 100 molecules are filling a volume of one nanometer length of the tube. The same number is obtained, if one is using the molecular density of dye crystals and the volume of the tube wall as obtained from cryo-TEM. X-ray crystal structure analysis was obtained by G. Reck et al. for millimeter sized single crystals of $\mathrm{C} 8 \mathrm{O} 3$ grown from DMSO solutions [65].

The fluorescence behavior of the tubular aggregates is also well explained by the bilayer tube model. It is assumed that the excitonic transitions of the two tubes can be considered as independent, that is, they have different ground states. However, because the lowest energy transition is different for the two layers, energy transfer occurs into the state with the lowest level. Therefore, fluorescence emission is observed from the two exciton states that are polarized parallel to the long axis of the tubules, but with an intensity ratio that may be different from that of the absorption, as it is observed in the fluorescence spectra of, for example, C8S3 in Figure 5. The double layer model allows to assign the observed emission peaks to the inner and outer cylinder, respectively. In the case of the model parameters given above, the energy transfer would be oriented from the outer cylinder into the inner cylinder. More details about the exciton dynamics and fluorescence properties of tubular aggregates are found in the contribution by A. Pugzlys in [56].

\subsection{Ribbon-like aggregates}

A second morphology that is frequently observed for the amphiphilic dye molecules 2 is a ribbon-like motif which is built by planar bilayer structures. This structure type was first observed for the carboxylic substituted dye $\mathrm{C} 8 \mathrm{O} 4$ [37] but it was also found for the sulfonic substituted dye C8S2 [44]. Typical cryo-TEM images of different types of ribbon-like structures are presented in Figure 7.

The structure of $\mathrm{C} 8 \mathrm{O} 4$ is shown in Figure 7(a). Straight objects with a striped pattern are visible that extend over several hundred nanometers in length and are of homogeneous thickness. These structures were interpreted as stacks of planar ribbons of dye bilayers. The stacking of ribbons becomes visible by the stripe pattern that is seen in a side view of the aggregate. A more detailed analysis of this structure is possible because some of the stacked ribbons are distorted. In that case, a typical modulation of the stripe appears in the transmission electron micrographs which could be modeled by computer graphics. In Figure $7(\mathrm{c})$, the planar aggregates are represented by grey shaded sheets that are twisted along the long axis of the aggregate. As in the case of the tubular aggregates, the transmission image of cryo-TEM was imi-

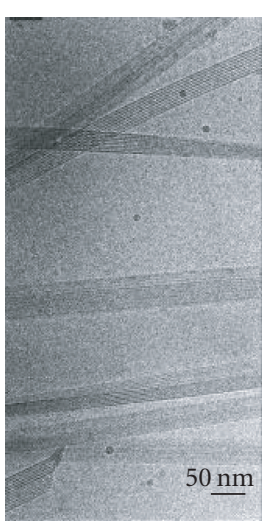

(a)

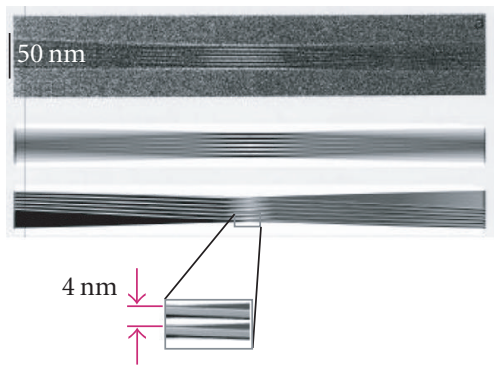

(c)

Figure 7: Cryo-TEM images of aggregate structures of (a) C8O4 $\left(3 \times 10^{-4} \mathrm{moll}-1\right.$ in $\left.1 \mathrm{mM} \mathrm{NaOH}\right)$ and (b) $\mathrm{C} 8 \mathrm{~S} 2\left(4 \times 10^{-4} \mathrm{M}\right.$ in $16 \mathrm{wt} \% \mathrm{MeOH})$. In (c), a magnified view of a laminar structure of (a) is shown. The original cryo-TEM image is compared to a computer graphic model. The model is built from a stack of ribbons and the whole stack is twisted. A transmission image is calculated assuming transparent material as in Figure 1. (Adapted with permission from [37, 44]. Copyright (2000) and (2004), American Chemical Society, resp.).

tated by calculating the projection of the computer graphic image assuming semitransparent objects. The resulting images reproduce the main features of the original image as can be seen in Figure $7(\mathrm{c})$. For the single ribbons, a thickness of approximately $4 \mathrm{~nm}$ is measured from the images, which confirms the assumption of a bilayer configuration of the $\mathrm{C} 8 \mathrm{O} 4$ molecules similar to a lipid bilayer.

Under certain conditions C8S2 also forms ribbon-like structures as presented in Figure 7(b). Here the structure of planar bilayers is less obvious but can be concluded from the folded object in the middle of Figure $7(\mathrm{~b})$. The stripes are interpreted again as signatures of the bilayers, seen edge-on [44]. But the most convincing indication for a ribbon-like structure is obtained from the optical spectra.

In Figure 8, the absorption spectra of C8O4 (a) and C8S2 (b) are presented. In case of $\mathrm{C} 8 \mathrm{O} 4$, the fluorescence is additionally shown. The absorption spectrum of the ribbon-like structures of $\mathrm{C} 8 \mathrm{O} 4$ exhibits the classical J-aggregate feature, 


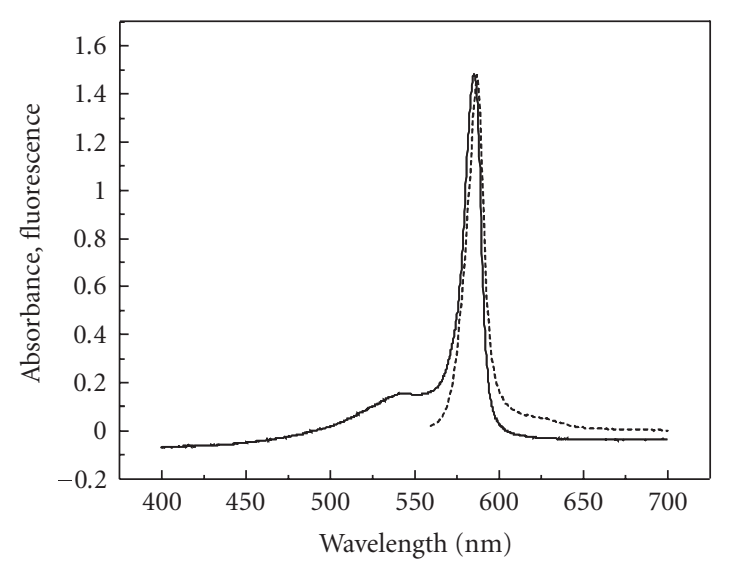

(a)

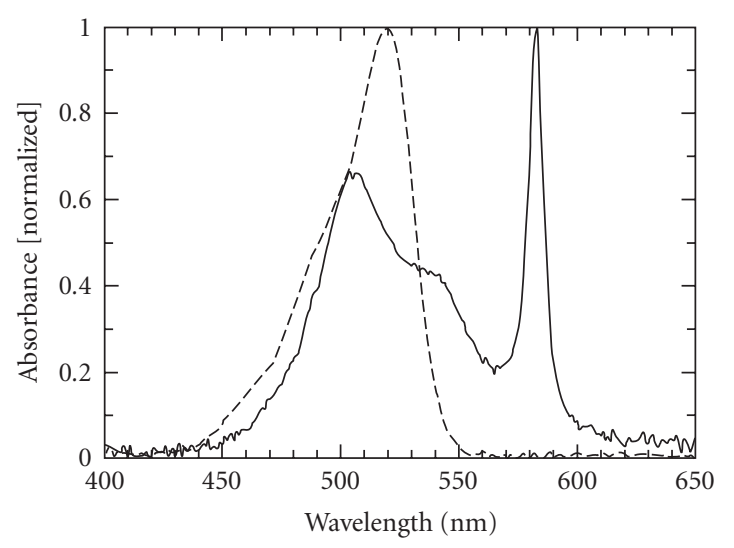

(b)

Figure 8: (a) Absorption (solid line) and fluorescence (dashed line) spectra of a $\mathrm{C} 8 \mathrm{O} 4$ solution. (b) Absorption spectrum of the ribbonlike structure of C8S2 shown in Figure 7. The monomer spectrum is indicated by the dashed line. (Adapted with permission from [44]. Copyright (2004) American Chemical Society.)

that is, one single narrow red-shifted J-band, indicating a two-dimensional brickwork-like arrangement. It seems that the number of multilayers within the stack does not have an effect on the spectra. This optical isolation of single bilayer sheets is explained by the distance caused by the enclosed hydrophobic chains. Similar spectra were also observed for other dyes [40]. For example, a single J-band was observed for dyes $\mathrm{CxO} 3$ with $x<7$ and $\mathrm{C} 8 \mathrm{O} y$ for $y=4$ and 5. However, an extended and systematic investigation of the structures is missing. The spectrum of C8S2 was interpreted as an exciton spectrum that is split almost symmetrically into two transitions (Davydov-splitting). This type of spectrum is a typical signature of a planar herringbone-like structure with two dyes per unit cell. Such spectra were found for many twodimensional systems investigated by the Langmuir-Blodgett technique [66, 67], but also for J-aggregates in aqueous solutions for a similar carbocyanine dye $[68,69]$.

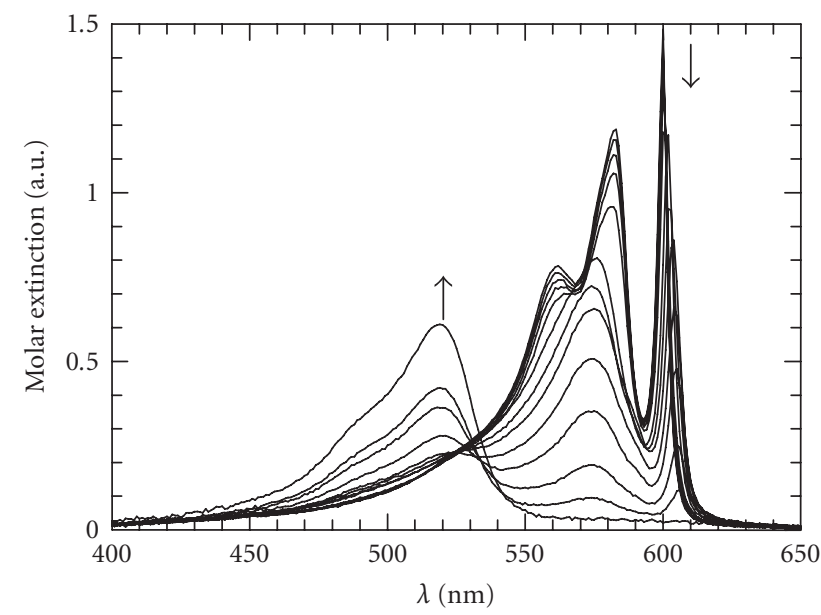

FIGURE 9: Absorption spectra of a solution of $\mathrm{C} 8 \mathrm{O} 3$ upon addition of $\mathrm{MeOH}$. Arrows indicate increasing amount of $\mathrm{MeOH}$ starting from 0 vol $\%$ to $34.4 \mathrm{vol} \%$. (Adapted with permission from [51]. Copyright (2002) American Chemical Society.)

\section{INFLUENCE OF ADDITIVES ON AGGREGATE STRUCTURES}

A remarkable sensitivity of the structure and morphology of the aggregates on addition of alcohols and other additives to the solvent was observed. Partially this can be understood because the structure of the aggregates is stabilized by hydrogen bonds and the hydrophobic effect which are known to be sensitive to the properties of the aqueous solvent [38].

\subsection{Solubility and addition of alcohol}

In general, the dyes 2 are soluble in aqueous solutions and aggregation is observed already at rather low concentrations, typically at $10^{-6} \mathrm{~mol} \mathrm{l}^{-1}$. For the carboxylic compounds, solubility and the aggregation behavior has to be controlled by addition of $\mathrm{NaOH}$ in a concentration range of $0.01 \mathrm{M}$ to $1 \mathrm{mM}$. Pure monomeric solutions at high concentration are possible in alcohol or dimethylsulfoxide (DMSO). The growth of macroscopic crystals of the dyes $\mathrm{C} 8 \mathrm{O} 3$ and $\mathrm{C} 8 \mathrm{O} 4$ was succeeded by cooling a high concentrated DMSO solution $[27,65]$.

The addition of any alcohol (EtOH, $\mathrm{MeOH}$, etc.) was found to have a major influence on the aggregate structure as seen by the optical spectra. For some dyes, even small amounts of less than $10 \mathrm{vol} \%$ of $\mathrm{MeOH}$ are sufficient to significantly change the aggregate structure. [51]. The change of the absorption spectrum upon addition of $\mathrm{MeOH}$ to an aqueous solution is shown in Figure 9. The absorption spectrum of the pure water solution exhibits three resolved absorption peaks as it was presented in Figure 4, but changes to a spectrum with only two resolved peaks at high $\mathrm{MeOH}$ concentration. For a $\mathrm{MeOH}$ content above $30 \mathrm{vol} \%$, the aggregates dissolve into monomers. Additionally, the intensity of the circular dichroism spectra increases by one to two orders of magnitude upon addition of alcohol to aqueous solutions. This effect is discussed in Section 5. 
The study of the influence of alcohol is of great importance, because dye solutions usually are prepared from high concentrated alcoholic stock solutions. This way of preparation, where a stock solution in the range of $10^{-3}$ to $10^{-2} \mathrm{M}$ is diluted with $\mathrm{NaOH}$ solution, is termed the "alcoholic route." For example, if this alcoholic route is selected to prepare $\mathrm{C} 8 \mathrm{O} 3$ solutions then an absorption spectrum with two resolved peaks may arise, or at least an intermediate spectrum of Figure 9. In most of the literature before 2002, solutions prepared by the alcoholic route were used, if not mentioned otherwise. Another way of preparation is the "direct route," where crystalline powder of the dye is added directly to $\mathrm{NaOH}$ solutions combined with stirring for at least 24 hours. In this case, the three-band absorption spectrum shown in Figure 9 is observed. Although it was speculated in [26] that the two spectra belong to different species of aggregates, it seems to be plausible from recent experiments that both spectra belong to a similar tubular structure. Because four transitions are necessary to describe the spectra of doublewall tubes, it is assumed that the number of peak maxima apparent in the absorption spectrum is lowered because of accidental degeneration.

It could be shown by means of cryo-TEM that the addition of alcohol to the $\mathrm{C} 8 \mathrm{O} 3$ solution does not significantly change the morphology of the tubes. However, the rope-like bundles of the tubes disintegrate into single tubes. The same effect was observed for the addition of poly(vinyl alcohol) (PVA) [52], where single tubules are found that are of nearly the same diameter as prepared without PVA. However, here the absorption spectrum has the same shape as the spectrum of a C8S3 solution prepared by the alcoholic route as presented in Figure 5.

It is a general tendency that addition of alcohols induces spectral changes but keeps the morphology unaffected. Obviously, alcohol groups are able to change the mutual orientation of the molecules which affects the energy levels of the excitonic transitions, but they are not capable of changing the tubular morphology which is stabilized by the hydrophobic chains.

The solubility conditions for the sulfonic compounds are more complicated. Whereas for example the compound TDBC (C2S4) is soluble in pure water up to rather high concentrations, the amphiphilic compound C8S2 is completely insoluble in pure aqueous solutions and has to be prepared by the alcoholic route [44]. Unlike with $\mathrm{C} 8 \mathrm{O} 3$, the addition of alcohol to C8S2 solutions changes not only the spectra but also the morphology of the aggregates. Tubular aggregates are obtained only for small dye concentrations and a low content of $\mathrm{MeOH}$, otherwise ribbon-like aggregates are found as depicted in Figure 7. These ribbon-like structures are not stable with respect to time but transform into tubular structures upon storage of the solutions for several weeks.

An even more complex behavior with respect to the solvent conditions is observed for the dye C8S3. In this case, tubular aggregates as shown in Figure 2 are found after preparation via the alcoholic as well as via the water route. They only differ in diameter, which is $17 \pm 0.2 \mathrm{~nm}$ when the dye was dissolved in pure water, while it was $13 \pm 0.2 \mathrm{~nm}$ for samples previously dissolved in methanol stock solutions. The aggregates formed in pure water can be converted into the other type by adding methanol if it exceeds more than approximately $18 \mathrm{wt} \%$. During days and weeks, the aggregates in pure water agglomerate into rope-like bundles while the aggregates in methanol/water mixtures transform into ribbon-like structures that are growing with time and are characterized by a new absorption spectrum. These ribbonlike structures are also in a metastable state in a sense that on time scales of several weeks or months they convert into giant tubes with diameters of up to $500 \mathrm{~nm}$ [45].

\subsection{Addition of surfactants}

It is well known that different surfactants are widely miscible in solution and often the mixtures self-assembled into aggregate structures which are different from the structures of the pure systems [38]. A similar effect was found for a mixture between TDBC (C2S4) and surfactants (N,N-dimethyln-alkyl betain) which caused a different aggregate structure of the TDBC as was seen by the optical spectra [53]. Therefore, it was obvious that the addition of surfactants could generate new aggregate structures for the amphiphilic dye molecules 2 as well. In detail, mixtures of two cosurfactants, the cationic TTAB (trimethyl tetradecyl ammonium bromide) and the anionic SDS (sodium dodecyl sulphate), with the dye molecules $\mathrm{C} 8 \mathrm{O} 3$ and $\mathrm{C} 8 \mathrm{O} 4$ were investigated $[47,48]$. The length of the alkyl chain of the surfactants was selected to fit approximately to the length of the octyl chains plus the width of the chromophore unit of the dye molecules.

In Figure 10, the influence of these two surfactants on the structures of aggregates of $\mathrm{C} 8 \mathrm{O} 3$ and $\mathrm{C} 8 \mathrm{O} 4$ is summarized. The amount of surfactant added was usually equimolar to the amount of dye molecules. For the dye $\mathrm{C} 8 \mathrm{O} 3$, which forms in the native state bundles of tubules, a twofold effect is observed for the addition of anionic or cationic surfactants (top row). The addition of anionic SDS leads to the formation of single tubules with an increased diameter (from $11 \mathrm{~nm}$ for $\mathrm{C} 8 \mathrm{O} 3$ in pure aqueous solution to $15 \mathrm{~nm}$ ). Upon storage for several days or weeks, the tubules are transformed into large multilamellar tubes. These multilamellar tubes look like as if the two-dimensional bilayer has been rolled up multiple times (cf. Figure 6). AFM images of these aggregates give the impression of "cigars" [47]. By small angle neutron scattering (SANS) on these cigar-like structure using deuterated SDS, it was proven that the surfactant molecules are really inserted into the aggregate structure. The insertion is probably forced by the hydrophobic interaction and the surfactant is located with its alkyl chain between the chains of the dyes. The additional alkyl chains stiffen the bilayer structure which explains the expansion of the tube diameter. Nevertheless, the exciton delocalization is maintained even in these structures. Their absorption spectra exhibit a broad J-band which might be caused by superposition of various spectra of tubes with different diameter.

The addition of the cationic TTAB leads to the formation of vesicular structures three days after preparation of the solution (cf. Figure 10). In the cryo-TEM images, unilamellar 


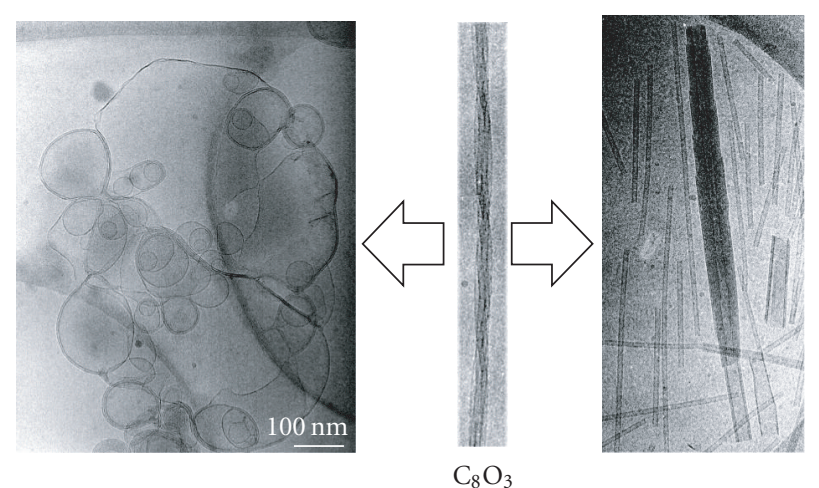

(a)

TTAB

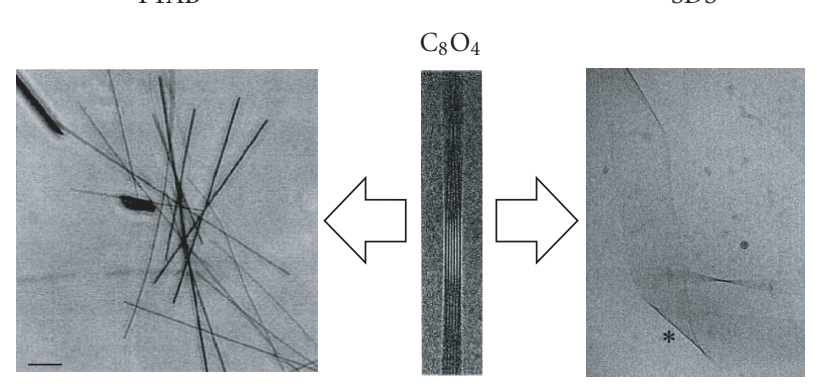

(b)

FIGURE 10: Overview of possible morphological changes of the J-aggregates of two slightly different amphiphilic cyanine dye molecules upon addition of a cationic (TTAB) and anionic (SDS) surfactant. The image at lower left corner is taken from optical microscopy (scale bar $10 \mu \mathrm{m}$ ), the other images are obtained from cryo-TEM (scale bar as indicated in the image). (Adapted with permission from [37, 47, 48]. Copyright (2000) and (2002), American Chemical Society, resp.).

vesicles with diameters between 40 and $100 \mathrm{~nm}$ are observed where the membrane has the typical thickness of $4 \mathrm{~nm}$. But also multilamellar vesicles and very large vesicles are found. The shape of the vesicles is superimposed by undulations which indicates a high flexibility of the bilayer membrane. The optical spectrum of the vesicular structure is broad and unstructured with almost no effect of J-aggregation which indicates an amorphous arrangement of the dyes. It seems that the opposite charge of the surfactant, on the one hand, destroys the molecular order of the dye bilayer and, on the other hand, reduces the net surface charge which increases the flexibility of the membrane.

The stabilization effect of the membrane due to anionic surfactants is also observed for the dye $\mathrm{C} 8 \mathrm{O} 4$. However, here it leads to a separation of the original stack of the bilayers into isolated single ribbons, which are more extended in width but of same thickness like a singular bilayer [48], see Figure 10. For mixture with the cationic surfactant TTAB the aggregates start to form macroscopic needle-like crystallites. The crystallization obviously is facilitated by the oppositely charged surfactant.

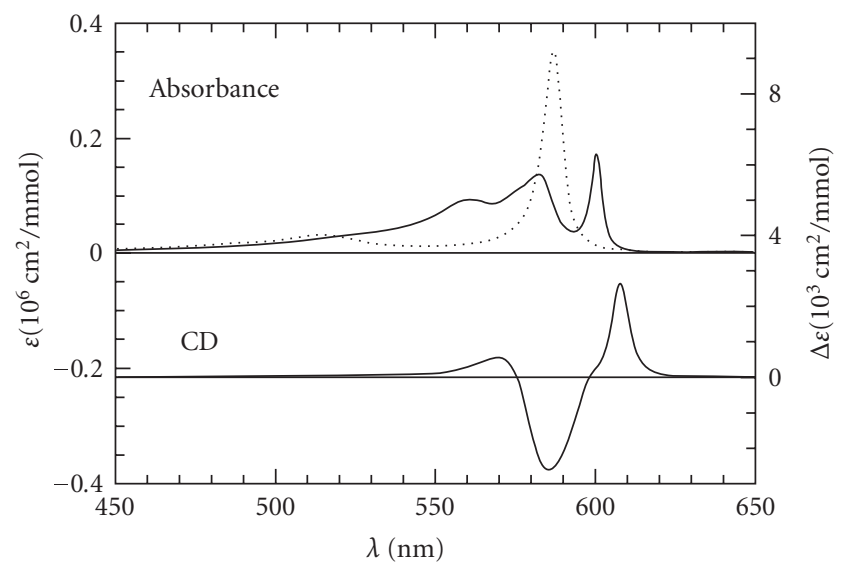

FIGURE 11: Absorption (left scale) and CD spectrum (right scale) of a $6.5 \times 10^{-6} \mathrm{M}$ solution of $\mathrm{C} 8 \mathrm{O} 3$, prepared by directly dissolving crystalline dye material in a $0.01 \mathrm{M} \mathrm{NaOH}$ solution. The absorption spectrum of achiral TDBC aggregates are shown by the dashed line as a reference. (Adapted with permission from [37]. Copyright (2000) American Chemical Society.)

The different behavior of $\mathrm{C} 8 \mathrm{O} 3$ and $\mathrm{C} 8 \mathrm{O} 4$ is remarkable, because the only difference between these two molecules is one carbon atom inserted in each spacer group at the $3,3^{\prime}$ position of the dyes. Therefore, one has to be aware that the presentation of Figure 10 is just an example of two of the dyes 2 , and no prediction can be made about the behavior of the other dyes under different solvent conditions or addition of other entities. A systematic investigation and a respective understanding of the intermolecular interactions are still missing.

\section{CHIRALITY}

One of the most surprising findings was the discovery that the tubular aggregates of the amphiphilic dyes 2 exhibit optical activity as measured by circular dichroism (CD) $[40$, 70]. A typical CD spectrum together with the absorption spectrum is shown in Figure 11 for C8O3. Since a CD effect is measurable for all those dyes that show spectra typical for tubular aggregates, it seems to be likely that all tubular aggregates of the amphiphilic dyes are chiral and more or less optically active. One has to emphasize that the occurrence of optical activity can be explained only by the assumption that the enantiomeric symmetry is broken. However, the analysis of TEM micrographs revealed that in any solution of $\mathrm{C} 8 \mathrm{O} 3$, right- and left-handed aggregates are present in approximately identical fraction (cf. Figure 12). Therefore, one has to assume that the CD signal is caused by a slight excess of one of the enantiomers. The large fluctuation of the CD intensity seen for different samples of $\mathrm{C} 8 \mathrm{O} 3$ strongly supports the assumption of an unspecified enantiomeric excess. It was proven by aggregates that were embedded into a solid film of poly(vinyl alcohol) and oriented by stretching the film that the CD spectra are not an artificial linear dichroism caused 


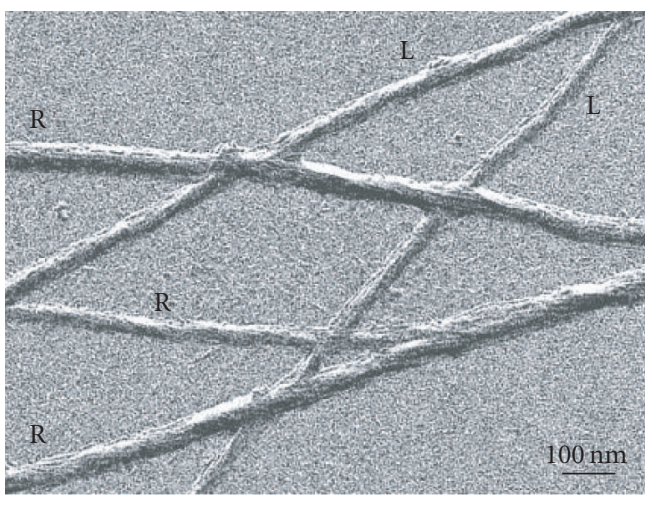

FIGURE 12: TEM micrograph of helical C8O3 aggregates after drying on a solid substrate and oblique shadowing with $\mathrm{Pt} / \mathrm{C}$. A $3 \times$ $10^{-4} \mathrm{M}$ solution was used and rope-like structures with left $(\mathrm{L})$ - and right $(\mathrm{R})$-handed twist are seen. (Adapted with permission from [71]. Copyright (2003) American Chemical Society.)

by accidental orientation in the solution. Up to date the reason for the symmetry break is not understood, but it seems to be a typical feature of the tubular aggregates.

The formation of chiral aggregate structures was reported before for other systems. However, they were either grown on chiral templates such as DNA [72], or chirality was induced by external stimulus [73] or due to chirality of the molecules [74]. It is known, for example, that PIC molecules in solution are chiral due to a twist of the two quinoline-rings against each other which is either right- or left-handed [75]. The growth of helical aggregates was proposed for these molecules [31]. However, it is seen by cryo-TEM images and electron diffraction [76] that these molecules form threadlike aggregates with a diameter of $2.3 \mathrm{~nm}$. The molecules are arranged in a $1: 1$ ratio of left-and right-handed twist giving a symmetric racemic mixture.

For the tubular aggregates, it was shown in Section 3 how one can understand that achiral molecules can be arranged on a cylindrical surface following a helix that may be left- or right-handed. Within the framework of this model, one can describe the features of the circular dichroism. The CD spectrum results from the chiral arrangement of the transition dipoles and not from an intrinsic chirality of the molecules. This type of CD effect is described in the literature as excitonic chirality [77], however, mostly it is applied to dimers. It is clear that the two pairs of optical transitions needed to describe the absorption spectra are both forming a couplet of a positive and negative signal in the $\mathrm{CD}$ spectrum. It is a peculiarity of the CD that the intensity of the positive and negative signal within one couplet must be identical which gives a strict condition for modeling the CD spectrum. Without prove, we want to mention that the $\mathrm{CD}$ spectrum presented in Figure 11 can be modeled by superposition of such two couplets.

The reason for the occurrence of optical activity due to enantiomeric excess of left- or right-handed helices is not understood. One explanation could be given based on nucleation and growth kinetics of the aggregates. It is known from nuclear magnetic resonance (NMR) experiments that the dyes in aqueous solutions exist as different conformers [77], where some of them are twisted with respect to the aromatic rings which induces chirality to the structure of the molecules. Both types of handedness are expected to be present of same quantity. It is probable that the first nuclei of aggregation are always dimers of two molecules of same handedness. During growth of the aggregates the handedness is prevailed. Because larger aggregates may break into peaces that again can grow, any small excess of one type of handedness is amplified to a measurable amount. For such a situation, one expects that the handedness of the excess varies statistically in a set of separately prepared solutions. Such a behavior, that is, statistical fluctuation of sign and magnitude of the $\mathrm{CD}$ signal was indeed observed for a large series of $\mathrm{C} 8 \mathrm{O} 3$ solutions [70]. In later experiments, this symmetric distribution could not be reproduced unambiguously. Merely, a spontaneous symmetry break was observed, not only in one solution, but also on the average of many solutions, which cannot be satisfactorily explained yet [65].

As for any chiral assembly, it was a challenging task to find a method to control the enantioselective growth of the helical tubular aggregates. Usually this is achieved by addition of chiral entities. Here it was found that the addition of achiral alcohols strongly enhances the CD signal, but does not allow to control the handedness. However, the sense of the handedness can be controlled using long chain chiral alcohols, such as (R)-2-octanol and (S)-2-octanol [71]. The addition of these substances has the same effect on the absorption spectra as the addition of $\mathrm{MeOH}$, see Figure 13, but the sign of the $\mathrm{CD}$ signal can be tuned according to the handedness of the alcohol. For the left-handed (S)-2-octanol, the $\mathrm{CD}$ band at $610 \mathrm{~nm}$ becomes negative while it is positive for (R)-2-octanol. A correlation between the different sign of the CD signals and the handedness of the rope-like aggregates was indeed found [71]. The (R)-2-octanol produces left-handed superhelices and vice versa. If one assumes that the chirality of the aggregates after addition of (R)-2-octanol is right-handed, than the handedness of a single tube is opposite to that of the bundle. Such an alternation of handedness for the binary, tertiary, and higher superstructure is well known for macroscopic ropes and may give a minimum in elastic energy.

\section{EXCITON DYNAMICS, SUPERQUENCHING, AND ELECTRON TRANSFER REACTIONS}

The outstanding optical properties of J-aggregates are caused by coherently coupled excited states, the molecular excitons. In a simple picture, such an exciton state can be considered as one huge molecule having a giant transition dipole moment and respective strong absorption coefficient for light. Due to static and dynamic molecular disorder, the extension of the exciton and hence the size of the dipole moment is restricted to a finite number $N_{\text {eff }}$ of coupled molecules. Therefore, the radiative lifetime $\tau^{0}$ of such supramolecules consisting of $N_{\text {eff }}$ single molecules should be strongly decreased as compared to their monomeric counterparts. For linear J-aggregates, the 


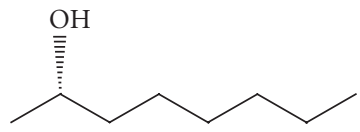

(S)

(a)

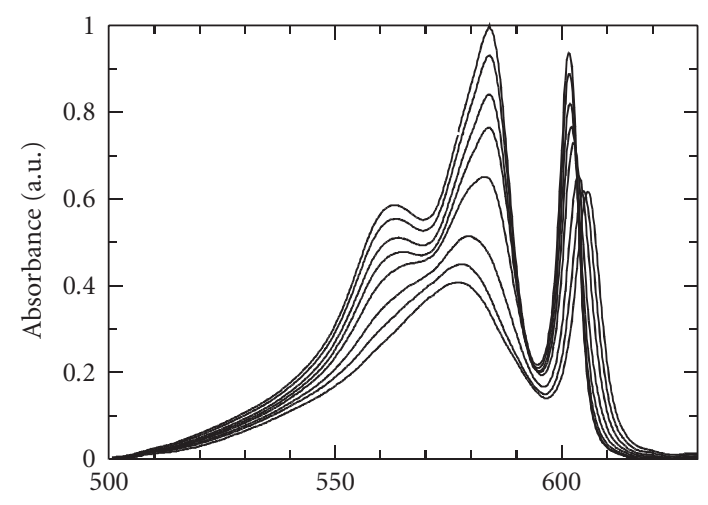

(b)

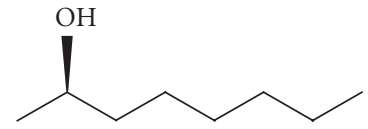

(R)

(c)

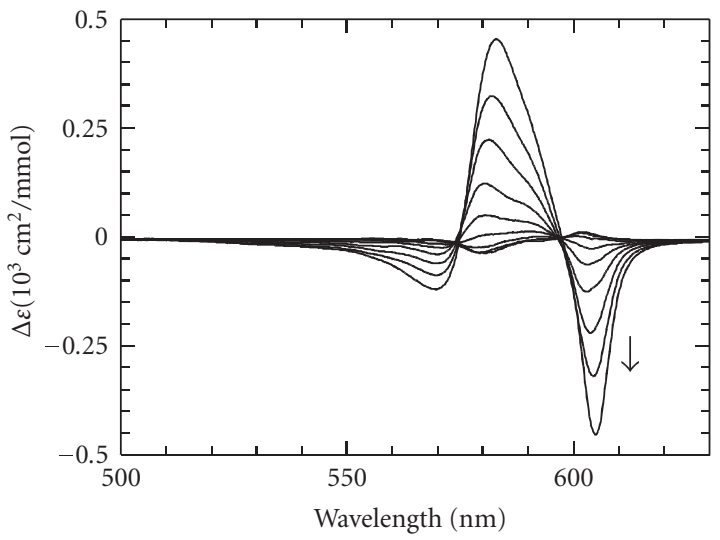

(d)

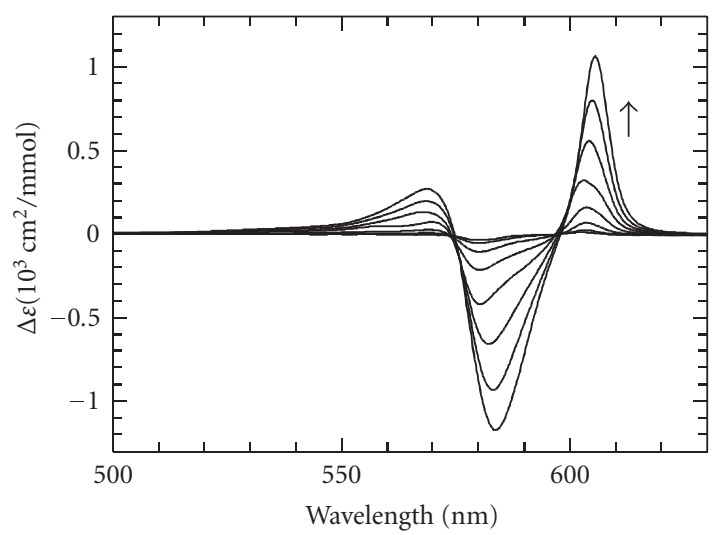

(e)

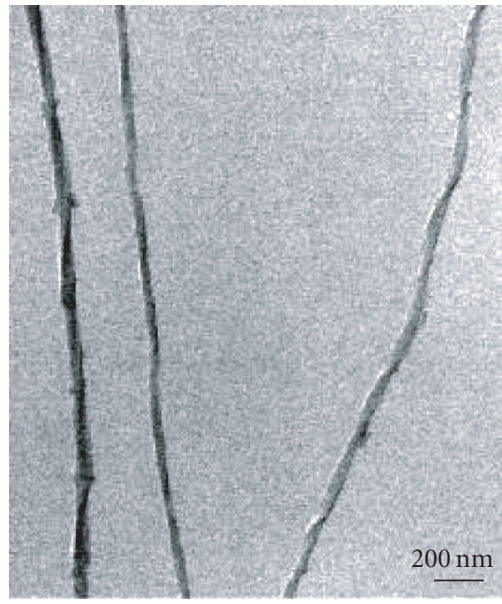

Right handed

(f)

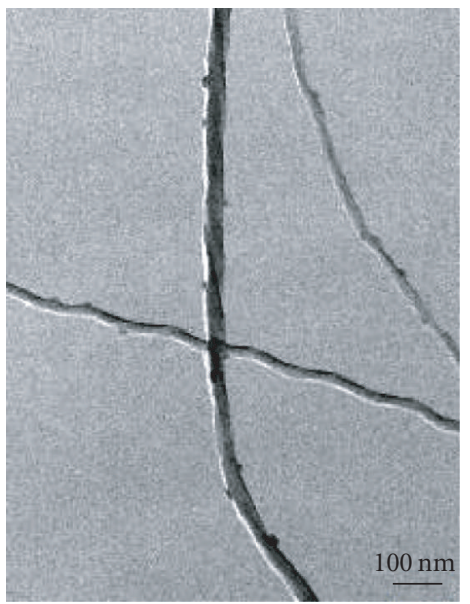

Left handed

(g)

FIGURE 13: Scheme demonstrating the effect of (a) left (S)- and (c) right (R)-handed octanol on the spectra and morphology of the aggregates of C8O3. In (b) the change of absorption spectrum and in (d) and (e) the increase of respective CD signal upon addition of octanol is shown. The molar ratio of octanol:dye was in the range of $0: 1$ to $24: 1$ for (S)- and $58: 1$ for (R)-octanol. TEM micrographs of aggregates after shadowing with Pt/C are presented in (f) and (g). An excess of right-handed structures are visible for the case of added (S)-octanol and vice versa. (Adapted with permission from [71]. Copyright (2003) American Chemical Society.) 
radiative lifetime is given by [78] $\tau_{(J)}^{0} \cong \tau_{(M)}^{0} / 0.81 \cdot N_{\text {eff }}$, where the indices $(J)$ and $(M)$ describe the radiative lifetime of the J-aggregates and monomers, respectively. In principle, it should be possible to determine the number of the coherently coupled molecules $N_{\text {eff }}$ from this equation by measuring the fluorescence lifetime and fluorescence quantum yield of the J-aggregates and the monomeric dyes. Many papers have reported on a shortening of the fluorescence lifetime of the PIC J-aggregates down to few picoseconds when time-resolved laser spectroscopy was applied [79]. However, later it turned out that the measurements were falsified by exciton-exciton annihilation processes that shorten the fluorescence lifetime significantly in J-aggregates even at relatively low laser intensities [80-82].

Only in 1991 coherent exciton migration has been definitely proven for PIC J-aggregates by measuring the temperature dependence of the fluorescence lifetime as well as through accumulated photon-echo and two-color pump-probe experiments [83-86]. It was found that for J-aggregates the fluorescence lifetime decreases for decreasing temperatures $[43,83]$, indicating that the lower the temperature the greater the number of coherently coupled molecules $N_{\text {eff }}$ and hence, the delocalization length of the excitons. Recently, a quantitative theoretical model for this behavior was presented [85].

From two-color pump-probe experiments, it followed that the delocalization length of the excitons in PIC J-aggregates amounts to $70 \pm 20$ molecules at $1.5 \mathrm{~K}$ [87]. Under the same conditions, values between 30 and 45 molecules were determined for the TDBC (C2S4) J-aggregates [88], whereas for the tubular aggregates of $\mathrm{C} 8 \mathrm{O} 3$ a delocalization size of approximately 95 molecules was measured at $1.5 \mathrm{~K}$ [55]. In case of TDBC (C2S4) J-aggregates, the exciton delocalization length was also determined at room temperature by femtosecond nonlinear optical experiments giving a value of 16 molecules [89].

Comprehensive theoretical work is necessary for a better understanding of the rather complicated exciton dynamics in tubular J-aggregates of the amphiphilic dye molecules. Numerous results have been collected by laser spectroscopic measurements which not only made possible the determination of the coherent delocalization lengths, but also gave insight in the radiative and thermal dephasing processes, the effect of excition-exciton annihilation, the influence of disorder, the meaning of intersite correlation processes, and the kinetics of intraband relaxation processes within the exciton band [55, 84, 90-93]. Most of these measurements have been made on aggregates of $\mathrm{C} 8 \mathrm{O} 3$, where the excitonic spectrum is less elaborate than it is in the case of the dye C8S3. Aggregates of the latter dye molecule show higher degree of molecular order which might result in larger exciton delocalization. Hence, it would be worthwhile to repeat some of the dynamical measurements on this system. Since the theory of optical dynamics in tubular J-aggregates will be reviewed by Knoester in [57], it should be only mentioned that the extension of the exciton theory to tubular molecular aggregates and especially to chiral helices [5962] allows a consistent interpretation of numerous experimental results as well as a development of precise mod- els of the shape and architecture of the tubular aggregates [50].

Besides exciton delocalization, fast migration of excitons within the aggregate occur by both coherent and incoherent energy transfer processes. The first description of this behavior was given by Scheibe [23]. He added small quantities of molecules that are able to quench the fluorescence emission to a solution of PIC J-aggregates and found that one quencher molecule per several ten thousand dye molecules was enough to reduce significantly the fluorescence intensity. In order to explain this finding, it was assumed that the excitons are moving across the aggregate over thousands of molecules during their lifetime until they are trapped by the quencher molecule. Later, this behavior was observed not only in dye aggregates [94] but also in conjugated polymers [95] and named as "superquenching."

The effect of exciton migration was also studied by measuring the rates of exciton-exciton annihilation in Jaggregates of PIC [82], the dye 3, $3^{\prime}$-disulfopropyl-5, $5^{\prime}$ dichloro-9-ethylthiacarbocyanine (THIATS) $[96,97]$, and the dye $\mathrm{C} 8 \mathrm{O} 3$ [98]. The dependence of fluorescence lifetime and fluorescence quantum yield on the excitation intensity was studied to obtain the rate of exciton migration or, in other words, the exciton mobility within the aggregates. From the mobility and the lifetime, a typical migration distance of the order of 10-20000 molecules could be estimated [82]. It was reported in [97] that the exciton mobility has a strong but nonmonotonous temperature dependence which was explained by different mechanisms of exciton transport at different temperature regimes. Efficient exciton-excitonannihilation was found for even very low temperature $(T<$ $20 \mathrm{~K})$ [98] and at higher temperatures $(T>110 \mathrm{~K})$. For low temperatures, it was suggested that exciton motion is due to temperature assisted hopping between coherent sites, while at high temperatures the motion was suggested to be dominated by incoherent scattering processes.

In order to study the exciton migration in tubular Jaggregates, the quenching experiments of Scheibe were repeated for aggregates of C8S3. In this case, an amphiphilic pyridine molecule was used which is well known to work as an electron acceptor and fluorescence quencher. The long alkyl chain supports the incorporation of the pyridine to the dye aggregates. A reduction of fluorescence intensity was found already at very low concentrations of the quencher as shown in Figure 14 by means of a double logarithmic SternVolmer plot.

In conventional systems, quenching is due to a bimolecular reaction between dye and quencher. For that case, the rate of quenching is proportional to the quencher concentration and the ratio between emission without $\left(I_{0}\right)$ and with quencher $(I)$ is given by $I_{0} / I=1+K_{\mathrm{SV}} \cdot$ [Quencher]. The Stern-Volmer quenching constant $K_{\mathrm{SV}}$ is obtained from the slope of a plot of $I_{0} / I$ versus quencher concentration. In the case of the J-aggregates of C8S3, the plot in Figure 14 is not linear, therefore a double logarithmic presentation was selected. Additionally, the quencher concentrations are very small compared to the dye concentration. Because of the nonlinearity of the quenching behavior not the quenching 


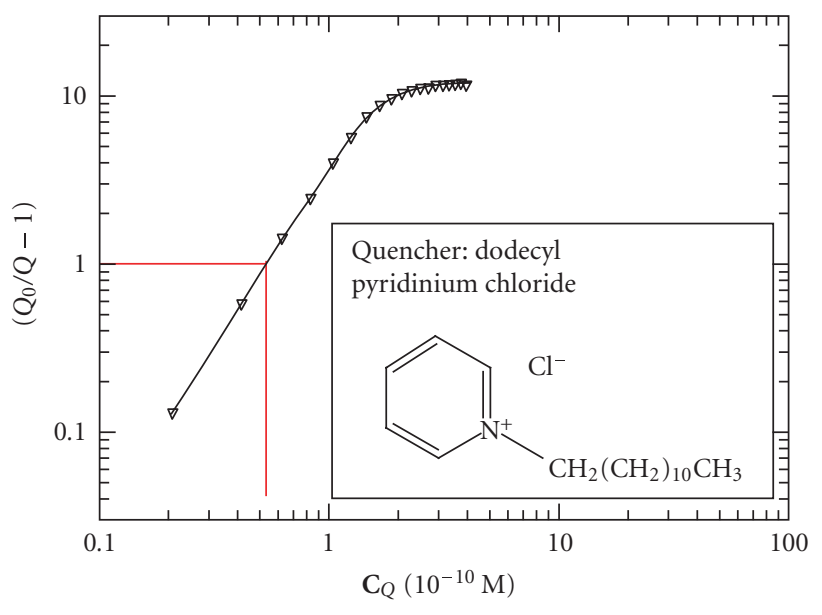

FIGURE 14: Double logarithmic presentation of a Stern-Volmer plot of fluorescence quenching of a C8S3 solution upon addition of dodecyl pyridine chloride. The concentration for $50 \%$ reduction in intensity is indicated.

constant $K_{\mathrm{SV}}$ but the number of quenchers per dye needed to reduce the initial emission intensity by $50 \%$ is used as a measure for the efficiency of the quenching process [94]. For the system C8S3/pyridine a ratio of 1 quencher per 5000 dyes is obtained as indicated in Figure 14. This value is very high compared to other dye systems and even larger than values obtained for conjugated polymers. If one converts this number into distances than one yields several ten to hundred nanometer as the expected distance of energy migration. Although the details of this quenching mechanism are not understood yet, this superquenching is a clear indication of efficient energy migration in the tubular J-aggregates. More experiments on fluorescence quenching are described by the contribution of Kirstein et al. in [99].

As mentioned in the introduction, exciton migration in J-aggregates is technically used in silver halide photography. However, there the mechanism is a priory rather complicated because PET reactions instantaneously compete with the intrinsic exciton migration. The dynamics of both processes depends on the type and size of the silver halide used as well as on the size of the J-aggregates and the dye coverage of the silver halide. Time-resolved fluorescence measurements at room temperature revealed time constants for the exciton migration and the subsequent electron transfer from Jaggregate into the silver halide of 100 and 700 femtoseconds, respectively [100-102].

The net reaction of spectrally sensitized PET in the photographic process is, simply spoken, injection of photoelectrons from excited dye molecules or dye aggregates into the silver halide's conduction band. There the silver ions are reduced to silver atoms which accumulate to latent silver specks. Afterwards the remaining electron-deficient dyes inject their positive hole into the silver halide's forbidden gap or valence band where the halogen ions are oxidized to halogen

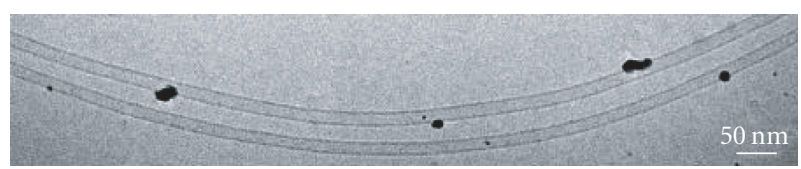

FIGURE 15: Cryo-TEM image of aggregates of C8S3 after addition of $\mathrm{AgNO}_{3}$ to the solution. The black spots are silver nanoparticles grown by photo-induced reduction of $\mathrm{Ag}^{+}$-ions.

which is deactivated by the environment. However, as mentioned already in the introduction, the reaction yield of PET reactions in such heterogeneous phases is strongly limited by the size of the interacting surface. Because the PET processes from J-aggregates into other species are of special interest for many applications, spectrally sensitized PET reactions in homogeneous phases are much more favourable.

A first example of spectrally sensitized PET application using J-aggregates of PIC (1) in aqueous solution has been described in 1993 [103, 104], where molecular hydrogen was generated by water cleavage with the help of J-aggregates of dye 1. This experiment was accomplished by Königstein et al. using PIC aggregates. As an electron acceptor viologen was used that was chemically bound to PIC (PIC-V). These PIC$\mathrm{V}$ molecules were intercalated by small amounts into the PIC aggregates [103, 104]. The supposed reaction scheme of the PET followed by cleavage of water in this system is briefly described as follows.

After photoexcitation of an aggregate, a delocalized exciton is formed which is rapidly trapped and localized at the PIC-V molecules where charge separation takes place to produce $\mathrm{PIC}^{+}-\mathrm{V}^{-}$. This state may relax back into the initial state, but with a certain probability the viologen is oxidized in order to reduce a proton into neutral hydrogen leaving a positively charged dye $\mathrm{PIC}^{+}-\mathrm{V}$. After reduction of hydrogen, the charged $\mathrm{PIC}^{+}$molecule is neutralized via an electron transfer from additional electron donating molecules in solution (EDTA in this case). By this reaction scheme, a mechanism is given where water is cleaved by light energy on cost of a reducing agent. However, the dye 1 seems to be not the best suited candidate for such an experiment. First, the dye is scarcely amphiphilic because of its less hydrophobic ethyl groups and missing hydrophilic substituents. Therefore, the structure of the J-aggregates is concentration dependent and ill defined $[35,36]$. Secondly, the low reduction potential of dye $1(-1.27$ Volt versus saturated calomel electrode (SCE), that is, $0.34 \mathrm{eV}$ lower than that of dye 2 [20]) is a poor precondition for an effective PET.

In recent experiments, a similar spectrally sensitized PET process was used to grow noble metal nanoparticles on the surface of J-aggregates by reduction of their respective metal ions in solution. In Figure 15, typical silver nanoparticles are shown on the tubular aggregates of C8S3. They are grown by addition of $\mathrm{AgNO}_{3}$ to the aggregate solution and illumination by room light. It is assumed that the reduction of the silver ions is realized by a similar reaction scheme as described above for the viologen. An electron transfer from the dye aggregate to the silver cation leads to neutral silver 
which crystallizes on the aggregates surface. The growth of only few and isolated Ag particles indicates a nucleation step which, however, could not be controlled. Although it cannot be proven unambiguously in the present state, it is assumed that this electron transfer is photo-assisted. Since in these experiments no electron donating molecules are present, some dyes at the aggregate surface must be oxidized. More details about this particle growth are found in a separate contribution by Kirstein et al. in [99].

\section{CONCLUSIONS AND OUTLOOK}

The main purpose of this contribution is to present some of the interesting features of amphiphilic cyanine dyes with respect to their aggregation properties. These dyes combine two important prerequisites for the built up of functional supramolecular complexes: first, the ability of surfactants to self-assemble into ordered structures; second, the possibility of closely packed cyanine dyes to form delocalized excited states due to the strong interaction between the transition dipoles in ordered molecular phases. Here, molecules built from 5, 5' , 6, $6^{\prime}$-tetrachlorobenzimidacarbocyanine dye with various hydrophobic and hydrophilic substituents at the $1,1^{\prime}$-and 3,3'-position are investigated. As could be shown by cryo-TEM investigations, these dyes form aggregates of various structures and morphologies depending on the structure of both the hydrophobic and hydrophilic substituents. The most interesting structural motif of these molecules that is found for many modifications of the chemical structure of the dyes and under different solvent conditions are three-dimensional tubes. The detailed structure of the tubes can be tuned and changed by addition of surfactants, alcohols, and other materials. It is even possible to add other molecules at the tubular aggregates such as poly(vinyl alcohol) or polyelectrolytes without interrupting the exciton delocalization. This opens the route to build hybrid systems of amphiphilic dye aggregates combined with other functional entities.

Due to the strong excitonic coupling, the aggregates of almost all different amphiphilic dyes show the behavior of Jaggregates, that means the ability of fast energy migration. The tubular structures have to be thought as a double-wall tube, where each tube consists of a brick work lattice of chromophores that is rolled up. Owing to the complicated arrangement of the molecules, the excitonic optical absorption spectra show not only one red-shifted band but several pronounced maxima. The spectra are very sensitive to structural changes of the aggregates and, therefore, any changes of structure due to external parameters such as the composition of the solvent or addition of surfactants can easily be monitored.

The delocalized excitons have the ability of efficient energy migration as shown by fluorescence quenching experiments. Additionally, photo-induced electron transfer (PET) from the aggregate to other species was demonstrated by reduction of silver ions. These properties make the J-aggregates of amphiphilic dye molecules very promising candidates for designing model systems of artificial light harvesting com- plexes (LHCs). These aggregates of water soluble amphiphilic cyanine dyes (amphipipes) are like a toolbox for the development of artificial LHCs, because they are easily available, the gain in free energy by an electron transfer reaction can be widely varied by their molecular structure, the architecture of their self-assembled structures can be controlled likewise by the structure of the constituent dyes as well as by the presence of surface-active additives, and they can be applied for electron transfer reactions in homogeneous (or at least microheterogeneous) phases making high reaction yields possible. Besides the chemistry to assemble artificial LHCs extensive static and time resolved spectroscopy will be necessary in order to gain deeper understanding of the exciton and charge transfer dynamics in those systems.

As the synthetic chemistry of water soluble amphiphilic cyanine dyes is only at its beginning stage, extensive future research work has to be done with respect to synthesis of structurally varied amphiphilic dyes. The important aspect of appearance of optical activity in this system must be taken into account. This is one of the rare systems that form chiral structures from achiral entities in a solution phase which also can easily be studied by optical spectroscopy in the visible range.

In summary, this new class of amphiphilic cyanine dyes provides a broad range of research fields, among which the investigation of spectrally sensitized PET reactions may be the most important and challenging task.

\section{ACKNOWLEDGMENTS}

The authors are grateful to their former and present coworkers (in alphabetical order): H.-W. Abraham, O. Al Khatib, H.von Berlepsch, C. Böttcher, C. Didraga, D. M. Eisele, J. Knoester, M. Lisk, J. Moll, A. Ouart, A. Pawlik, A. Pugžlys, G. Reck, U. De Rossi, and C. Spitz. They also thank M. Hennecke (Bundesanstalt für Materialforschung und -prüfung), H. Möhwald (Max-Planck-Institut für Kolloid-und Grenzflächenforschung, Potsdam), and J. P. Rabe (Humboldt Universität zu Berlin) for their continuous and generous support of this work. Financial support was obtained from grants of the Deutsche Forschungsgemeinschaft (DFG; Sfb 337 and Sfb 448, DA 268/6-1,2; AB 74/5-1,2,3; KI 544/1,2,3; and INTAS project 97-10434).

\section{REFERENCES}

[1] X. Hu, T. Ritz, A. Damjanović, and K. Schulten, "Pigment organization and transfer of electronic excitation in the photosynthetic unit of purple bacteria," The Journal of Physical Chemistry: B, vol. 101, no. 19, pp. 3854-3871, 1997.

[2] J. Deisenhofer and H. Michel, "Das photosynthetische Reaktionszentrum des Purpurbakteriums rhodopseudomonas viridis (Nobel-Vortrag)," Angewandte Chemie, vol. 101, no. 7, pp. 872-892, 1989.

[3] R. Huber, "Eine strukturelle Grundlage für die Übertragung von Lichtenergie und Elektronen in der Biologie (NobelVortrag)," Angewandte Chemie, vol. 101, no. 7, pp. 849-871, 1989. 
[4] G. Mc Dermott, S. M. Prince, A. A. Freer, et al., "Crystal structure of an integral membrane light-harvesting complex from photosynthetic bacteria," Nature, vol. 374, no. 6522, pp. 517-521, 1995.

[5] J. Koepke, X. C. Hu, C. Muenke, K. Schulten, and H. Michel, "The crystal structure of the light-harvesting complex II (B800-850) from Rhodospirillum molischianum," Structure, vol. 4, no. 5, pp. 581-597, 1996.

[6] T. Miyatake, H. Tamiaki, A. R. Holzwarth, and K. Schaffner, "Self-assembly of synthetic zinc chlorins in aqueous microheterogeneous media to an artificial supramolecular lightharvesting device," Helvetica Chimica Acta, vol. 82, no. 6, pp. 797-810, 1999.

[7] D.-J. Heijs, V. A. Malyshev, and J. Knoester, "Trapping time statistics and efficiency of transport of optical excitations in dendrimers," The Journal of Chemical Physics, vol. 121, no. 10, pp. 4884-4892, 2004.

[8] F. Voegtle, Ed., Dendrimers, Springer, Berlin, Germany, 1998.

[9] M.-S. Choi, T. Aida, T. Yamazaki, and I. Yamazaki, "A large dendritic multiporphyrin array as a mimic of the bacterial light-harvesting antenna complex: molecular design of an efficient energy funnel for visible photons," Angewandte Chemie, vol. 113, no. 17, pp. 3294-3298, 2001.

[10] N. Tomioka, D. Takasu, T. Takahashi, and T. Aida, "Elektrostatische aggregation von dendritischen elektrolyten: negativ und positiv geladene porphyrin-dendrimere," Angewandte Chemie, vol. 110, no. 11, pp. 1611-1614, 1998.

[11] G. Steinberg-Yfrach, J.-L. Rigaud, E. N. Durantini, A. L. Moore, D. Gust, and T. A. Moore, "Light-driven production of ATP catalysed by $\mathrm{F}_{0} \mathrm{~F}_{1}$-ATP synthase in an artificial photosynthetic membrane," Nature, vol. 392, no. 6675, pp. 479 482, 1998.

[12] J.-M. Lehn, Supramolecular Chemistry, Wiley-VCH, Weinheim, Germany, 1995.

[13] I. Yamazaki, N. Tamai, and T. Yamazaki, "Electronic excitation transfer in organized molecular assemblies," The Journal of Physical Chemistry, vol. 94, no. 2, pp. 516-525, 1990.

[14] M. Grätzel, "Photoelectrochemical cells," Nature, vol. 414, no. 6861, pp. 338-344, 2001.

[15] J. E. Maskasky, "Molecular orientation of individual J aggregates on gelatin-grown AgBr tabular microcrystals," Langmuir, vol. 7, no. 2, pp. 407-421, 1991.

[16] S. Kirstein, R. Steitz, R. Garbella, and H. Möhwald, "Herringbone structure in two-dimensional single crystals of cyanine dyes. I. Detailed structure analysis using electron diffraction," The Journal of Chemical Physics, vol. 103, no. 2, pp. 818-825, 1995.

[17] C. E. K. Mees and T. H. James, The Theory of the Photographic Process, Macmillan, New York, NY, USA, 3rd edition, 1966.

[18] S. Daehne, "Spectral sensitization and electronic structure of organic dyes," Photographic Science and Engineering, vol. 23, no. 4, pp. 219-239, 1979.

[19] T. Tani, T. Suzumoto, and K. Ohzeki, "Energy gap dependence of efficiency of photoinduced electron transfer from cyanine dyes to silver bromide microcrystals in spectral sensitization," The Journal of Physical Chemistry, vol. 94, no. 4, pp. 1298-1301, 1990.

[20] K. Hosoi, A. Hirano, and T. Tani, "Dynamics of photocreated positive holes in silver bromide microcrystals with adsorbed cyanine dyes," Journal of Applied Physics, vol. 90, no. 12, pp. 6197-6204, 2001.
[21] E. E. Jelly, "Spectral absorption and fluorescence of dyes in the molecular state," Nature, vol. 138, pp. 1009-1010, 1936.

[22] G. Scheibe, A. Mareis, and H. Ecker, "Über reversible polymerisation als ursache neuartiger absorptionsbanden (III)," Naturwissenschaften, vol. 25, no. 29, pp. 474-475, 1937.

[23] G. Scheibe, A. Schöntag, and F. Katheder, "Fluoreszenz und Energiefortleitung bei reversibel polymerisierten Farbstoffen," Naturwissenschaften, vol. 27, no. 29, pp. 499-501, 1939.

[24] S. De Boer and D. A. Wiersma, "Optical dynamics of exciton and polaron formation in molecular aggregates," Chemical Physics, vol. 131, no. 1, pp. 135-144, 1989.

[25] H. Kuhn, D. Möbius, and H. Bücher, "Spectroscopy of monolayer assemblies," in Physical Methods of Chemistry, A. Weissberger and B. Rossiter, Eds., pp. 577-578, Wiley-Interscience, New York, NY, USA, 1972.

[26] C. Spitz, J. Knoester, A. Ouart, and S. Daehne, "Polarized absorption and anomalous temperature dependence of fluorescence depolarization in cylindrical J-aggregates," Chemical Physics, vol. 275, no. 1-3, pp. 271-284, 2002.

[27] A. Pawlik, A. Ouart, S. Kirstein, H.-W. Abraham, and S. Daehne, "Synthesis and UV/Vis Spectra of J-aggregating $5,5^{\prime}, 6,6^{\prime}$-tetrachlorobenzimidacarbocyanine dyes for artificial light-harvesting systems and for asymmetrical generation of supramolecular helices," European Journal of Organic Chemistry, vol. 2003, no. 16, pp. 3065-3080, 2003.

[28] S. Daehne, "Color and constitution: one hundred years of research," Science, vol. 199, no. 4334, pp. 1163-1167, 1978.

[29] G. Bach and S. Daehne, "Cyanine dyes and related compounds," in RODD's Chemistry of Carbon Compounds. Vol. $I V$, M. Sainsbury, Ed., chapter 15, pp. 383-481, Elsevier Science, Amsterdam, The Netherlands, 2nd supplement to 2nd edition, 1997.

[30] T. Tanaka and T. Matsubara, "Concentration- and temperature-dependent size of metastable J-aggregate particles of cyanine dyes," Journal of Imaging Science and Technology, vol. 37, no. 6, pp. 585-588, 1993.

[31] E. Daltrozzo, G. Scheibe, K. Gschwind, and F. Haimerl, "On the structure of the J-aggregates of pseudoisocyanine," Photographic Science and Engineering, vol. 18, no. 4, pp. 441-450, 1974.

[32] S. Makio, N. Kanamaru, and J. Tanaka, "The J-aggregate $5,5^{\prime}, 6,6^{\prime}$-Tetrachloro-1, 1' -diethyl-3, 3' -bis(4-sulfobutyl) benzimidazolocarbocyanine sodium salt in aqueous solution," Bulletin of the Chemical Society of Japan, vol. 53, no. 11, pp. 3120-3124, 1980.

[33] L. Daehne, E. Biller, and H. Baumgärtel, "Polariton-induced color tuning of thin dye layers," Angewandte ChemieInternational Edition, vol. 37, no. 5, pp. 646-649, 1998.

[34] M. Li, A. F. Wang, G. Z. Mao, and L. Daehne, "Surfacedirected adsorption in the epitaxy growth of streptocyanine dye crystals," The Journal of Physical Chemistry: B, vol. 103, no. 50, pp. 11161-11168, 1999.

[35] H. von Berlepsch, C. Böttcher, and L. Daehne, "Structure of J-aggregates of pseudoisocyanine dye in aqueous solution," The Journal of Physical Chemistry: B, vol. 104, no. 37, pp. 8792-8799, 2000.

[36] H. von Berlepsch and C. Böttcher, "Network superstructure of pseudoisocyanine J-aggregates in aqueous sodium chloride solution revealed by cryo-transmission electron microscopy," The Journal of Physical Chemistry: B, vol. 106, no. 12, pp. 3146-3150, 2002. 
[37] H. von Berlepsch, C. Böttcher, A. Ouart, C. Burger, S. Daehne, and S. Kirstein, "Supramolecular structures of Jaggregates of carbocyanine dyes in solution," The Journal of Physical Chemistry: B, vol. 104, no. 22, pp. 5255-5262, 2000.

[38] D. F. Evans and H. Wennerström, The Colloidal DomainWhere Physics, Chemistry, Biology, and Technology Meet, Wiley-VCH, Weinheim, Germany, 2nd edition, 1999.

[39] U. De Rossi, J. Moll, M. Spieles, et al., "Control of the J-aggregation phenomenon by variation of the $N$-alkyl-substituents," Journal für Praktische Chemie/Chemiker-Zeitung, vol. 337, no. 1, pp. 203-208, 1995.

[40] A. Pawlik, S. Kirstein, U. De Rossi, and S. Daehne, "Structural conditions for spontaneous generation of optical activity in J-aggregates," The Journal of Physical Chemistry: B, vol. 101, no. 29, pp. 5646-5651, 1997.

[41] FEW Chemicals GmbH, Industriepark Wolfen-Thalheim, P.O.Box 1340, D-06756 Wolfen, Germany.

[42] J. Grad, G. Hernandez, and S. Mukamel, "Radiative decay and energy transfer in molecular aggregates: the role of intermolecular dephasing," Physical Review A, vol. 37, no. 10, pp. 3835-3846, 1988.

[43] H. Fidder, J. Knoester, and D. A. Wiersma, "Superradiant emission and optical dephasing in J-aggregates," Chemical Physics Letters, vol. 171, no. 5-6, pp. 529-536, 1990.

[44] H. von Berlepsch, S. Kirstein, and C. Böttcher, "Supramolecular structure of J-aggregates of a sulfonate substituted amphiphilic carbocyanine dye in solution: methanol-induced ribbon-to-tubule transformation," The Journal of Physical Chemistry: B, vol. 108, no. 48, pp. 18725-18733, 2004.

[45] H. von Berlepsch, C. Böttcher, and S. Kirstein, to appear in The Journal of Physical Chemistry.

[46] M. M. S. Abdel-Mottaleb, M. Van der Auweraer, and M. S. A. Abdel-Mottaleb, "Photostability of J-aggregates adsorbed on $\mathrm{TiO}_{2}$ nanoparticles and AFM imaging of J-aggregates on a glass surface," International Journal of Photoenergy, vol. 6, no. 1, pp. 29-33, 2004.

[47] H. von Berlepsch, C. Böttcher, A. Ouart, et al., "Surfactantinduced changes of morphology of J-aggregates: superhelixto-tubule transformation," Langmuir, vol. 16, no. 14, pp. 5908-5916, 2000.

[48] H. von Berlepsch, M. Regenbrecht, S. Daehne, S. Kirstein, and C. Böttcher, "Surfactant-induced separation of stacked J-aggregates. Cryo-transmission electron microscopy studies reveal bilayer ribbons," Langmuir, vol. 18, no. 7, pp. 29012907, 2002.

[49] H. Furhop and J. König, Membranes and Molecular Assemblies: The Synkinetic Approach, The Royal Society of Chemistry, Cambridge, UK, 1994.

[50] C. Didraga, A. Pugzlys, P. R. Hania, H. von Berlepsch, K. Duppen, and J. Knoester, "Structure, spectroscopy, and microscopic model of tubular carbocyanine dye aggregates," The Journal of Physical Chemistry: B, vol. 108, no. 39, pp. 14976-14985, 2004.

[51] H. von Berlepsch, S. Kirstein, and C. Böttcher, "Effect of alcohols on J-aggregation of a carbocyanine dye," Langmuir, vol. 18, no. 20, pp. 7699-7705, 2002.

[52] H. von Berlepsch, S. Kirstein, R. Hania, C. Didraga, A. Pugzlys, and C. Böttcher, "Stabilization of individual tubular Jaggregates by poly(vinyl alcohol)," The Journal of Physical Chemistry: B, vol. 107, no. 51, pp. 14176-14184, 2003.

[53] U. De Rossi, S. Daehne, and M. Lindrum, "Increased coupling size in J-aggregates through $N-n$-alkyl betaine surfactants," Langmuir, vol. 12, no. 5, pp. 1159-1165, 1996.
[54] C. Spitz, J. Knoester, A. Ouart, and S. Daehne, "Polarized absorption and anomalous temperature dependence of fluorescence depolarization in cylindrical J-aggregates," Chemical Physics, vol. 275, no. 1-3, pp. 271-284, 2002.

[55] S. S. Lampoura, C. Spitz, S. Daehne, J. Knoester, and K. Duppen, "The optical dynamics of excitons in cylindrical Jaggregates," The Journal of Physical Chemistry: B, vol. 106, no. 12, pp. 3103-3111, 2002.

[56] A. Pugzlys, P. R. Hania, R. Augulis, K. Duppen, and P. H. M. van Loosdrecht, "Cylindrical aggregates of 5, 5', 6, 6' tetrachlorobenzimida-carbocyanine amphiphilic derivatives: structure-related optical properties and exciton dynamics," International Journal of Photoenergy, vol. 2006, Article ID 29623, 9 pages, 2006.

[57] J. Knoester, "Modeling the optical properties of excitons in linear and tubular J-aggregates," International Journal of Photoenergy, vol. 2006, Article ID 61364, 10 pages, 2006.

[58] H. Kuhn and C. Kuhn, "Chromophore coupling effects," in J-Aggregates, C. Kobayashi, Ed., World Scientific, Singapore, 1996.

[59] C. Didraga and J. Knoester, "Absorption and dichroism spectra of cylindrical J-aggregates and chlorosomes of green bacteria," Journal of Luminescence, vol. 102-103, pp. 60-66, 2003.

[60] M. Bednarz and J. Knoester, "The linear absorption and pump-probe spectra of cylindrical molecular aggregates," The Journal of Physical Chemistry: B, vol. 105, no. 51, pp. 12913-12923, 2001.

[61] C. Didraga, J. A. Klugkist, and J. Knoester, "Optical properties of helical cylindrical molecular aggregates: the homogeneous limit," The Journal of Physical Chemistry: B, vol. 106, no. 44, pp. 11474-11486, 2002.

[62] C. Didraga and J. Knoester, "Exchange narrowing in circular and cylindrical molecular aggregates: degenerate versus nondegenerate states," Chemical Physics, vol. 275, no. 1-3, pp. 307-318, 2002.

[63] C. Böttcher, H. von Berlepsch, and S. Kirstein, unpublished results.

[64] V. Czikkely, H. D. Försterling, and H. Kuhn, "Extended dipole model for aggregates of dye molecules," Chemical Physics Letters, vol. 6, no. 3, pp. 207-210, 1970.

[65] S. Kirstein, H. von Berlepsch, C. Böttcher, et al., "Chiral Jaggregates formed by achiral cyanine dyes," Chemical Physics and Physical Chemistry, vol. 1, no. 3, pp. 146-150, 2000.

[66] S. Kirstein and H. Möhwald, "Herringbone structure in twodimensional single crystals of cyanine dyes. II. Optical properties," The Journal of Chemical Physics, vol. 103, no. 2, pp. 826-833, 1995.

[67] K. Saito, K. Ikegami, S.-I. Kuroda, Y. Tabe, and M. Sugi, "Formation of herringbone structure with davydov splitting in cyanine dye-adsorbed langmuir-blodgett films," Japanese Journal of Applied Physics, vol. 30, part 1, no. 8, pp. 18361840, 1991.

[68] D. M. Basko, A. N. Lobanov, A. V. Pimenov, and A. G. Vitukhnovsky, "Molecular arrangement of the Davydov-split dye aggregates," Chemical Physics Letters, vol. 369, no. 1-2, pp. 192-197, 2003.

[69] I. G. Scheblykin, M. M. Bataiev, M. Van der Auweraer, and A. G. Vitukhnovsky, "Dimensionality and temperature dependence of the radiative lifetime of J-aggregates with Davydov splitting of the exciton band," Chemical Physics Letters, vol. 316, no. 1-2, pp. 37-44, 2000. 
[70] U. De Rossi, S. Daehne, S. C. J. Meskers, and H. P. J. M. Dekkers, "Spontaneous formation of chirality in J-aggregates showing Davydov splitting," Angewandte Chemie International Edition in English, vol. 35, no. 7, pp. 760-763, 1996.

[71] H. von Berlepsch, S. Kirstein, and C. Böttcher, "Controlling the helicity of tubular J-aggregates by chiral alcohols," The Journal of Physical Chemistry: B, vol. 107, no. 36, pp. 96469654, 2003.

[72] M. Wang, G. L. Silva, and B. A. Armitage, "DNA-templated formation of a helical cyanine dye J-aggregate," Journal of the American Chemical Society, vol. 122, no. 41, pp. 9977-9986, 2000.

[73] J. M. Ribó, J. Crusats Sagues, F. Sagués, J. Claret, and R. Rubires, "Chiral sign induction by vortices during the formation of mesophases in stirred solutions," Science, vol. 292, no. 5524, pp. 2063-2066, 2001.

[74] A. E. Rowan and R. J. M. Nolte, "Molekulare Programmierung von Helicität," Angewandte Chemie, vol. 110, no. 12, pp. 65-71, 1998.

[75] B. Dammeier and W. Hoppe, "Die Kristall- und Molekülstruktur von $N, N^{\prime}$-diäthyl-pseudoisocyaninchlorid," Acta Crystallographica Section B, vol. 27, part 12, pp. 2364-2370, 1971.

[76] H. von Berlepsch, S. Möller, and L. Daehne, "Optical properties of crystalline pseudoisocyanine (PIC)," The Journal of Physical Chemistry: B, vol. 105, no. 24, pp. 5689-5699, 2001.

[77] R. Radeglia and S. Daehne, unpublished results.

[78] J. Grad, G. Hernandez, and S. Mukamel, "Radiative decay and energy transfer in molecular aggregates: the role of intermolecular dephasing," Physical Review A, vol. 37, no. 10, pp. 3835-3846, 1988.

[79] F. Fink, E. Klose, K. Teuchner, and S. Daehne, "Determination of the fluorescence decay time of the I-aggregates of pseudoisocyanine," Chemical Physics Letters, vol. 45, no. 3, pp. 548-549, 1977.

[80] D. V. Brumbaugh, A. A. Muenter, W. Knox, G. Mourou, and B. Wittmershaus, "Singlet exciton annihilation in the picosecond fluorescence decay of $1,1^{\prime}$-diethyl-2, 2' -cyanine chloride dye J-aggregate," Journal of Luminescence, vol. 31-32, part 2, pp. 783-785, 1984.

[81] H. Stiel, S. Daehne, and K. Teuchner, "J-aggregates of pseudoisocyanine in solution: new data from nonlinear spectroscopy," Journal of Luminescence, vol. 39, no. 6, pp. 351357,1988 .

[82] V. Sundström, T. Gillbro, R. A. Gadonas, and A. Piskarskas, "Annihilation of singlet excitons in J-aggregates of pseudoisocyanine (PIC) studied by pico- and subpicosecond spectroscopy," The Journal of Chemical Physics, vol. 89, no. 5, pp. 2754-2762, 1988.

[83] H. Fidder, J. Terpstra, and D. A. Wiersma, "Dynamics of Frenkel excitons in disordered molecular aggregates," The Journal of Chemical Physics, vol. 94, no. 10, pp. 6895-6907, 1991.

[84] H. Fidder, J. Knoester, and D. A. Wiersma, "Optical properties of disordered molecular aggregates: a numerical study," The Journal of Chemical Physics, vol. 95, no. 11, pp. 78807890, 1991.

[85] D. J. Heijs, V. A. Malyshev, and J. Knoester, "Decoherence of excitons in multichromophore systems: thermal line broadening and destruction of superradiant emission," Physical Review Letters, vol. 95, no. 17, Article ID 177402, 2005.

[86] H.-P. Dorn and A. Müller, "Scheibe-aggregates of Pseudoisocyanine in solution and in molecular monolayers," Applied Physics B: Lasers and Optics, vol. 43, no. 3, pp. 167-172, 1987.
[87] J. R. Durrant, J. Knoester, and D. A. Wiersma, "Local energetic disorder in molecular aggregates probed by the oneexciton to two-exciton transition," Chemical Physics Letters, vol. 222, no. 5, pp. 450-456, 1994.

[88] J. Moll, S. Daehne, J. R. Durrant, and D. A. Wiersma, "Optical dynamics of excitons in J-aggregates of a carbocyanine dye," The Journal of Chemical Physics, vol. 102, no. 16, pp. 63626370, 1995.

[89] M. van Burgel, D. A. Wiersma, and K. Duppen, "The dynamics of one-dimensional excitons in liquids," The Journal of Chemical Physics, vol. 102, no. 1, pp. 20-33, 1995.

[90] S. de Boer, K. J. Vink, and D. A. Wiersma, "Optical dynamics of condensed molecular aggregates: an accumulated photonecho and hole-burning study of the J-aggregate," Chemical Physics Letters, vol. 137, no. 2, pp. 99-106, 1987.

[91] H. Fidder and D. A. Wiersma, "Resonance-light-scattering study and line-shape simulation of the J band," Physical Review Letters, vol. 66, no. 11, pp. 1501-1504, 1991.

[92] F. C. Spano and S. Mukamel, "Superradiance in molecular aggregates," The Journal of Chemical Physics, vol. 91, no. 2, pp. 683-700, 1989.

[93] S. de Boer and D. A. Wiersma, "Dephasing-induced damping of superradiant emission in J-aggregates," Chemical Physics Letters, vol. 165, no. 1, pp. 45-53, 1990.

[94] L. Lu, R. Helgeson, R. M. Jones, D. McBranch, and D. Whitten, "Superquenching in cyanine pendant $\operatorname{poly}\left({ }_{L}-\right.$ lysine $)$ dyes: dependence on molecular weight, solvent, and aggregation," Journal of the American Chemical Society, vol. 124, no. 3, pp. 483-488, 2002.

[95] C. Fan, S. Wang, J. W. Hong, G. C. Bazan, K. W. Plaxco, and A. J. Heeger, "Beyond superquenching: hyper-efficient energy transfer from conjugated polymers to gold nanoparticles," Proceedings of the National Academy of Sciences of the United States of America, vol. 100, no. 11, pp. 6297-6301, 2003.

[96] I. G. Scheblykin, O. P. Varnavsky, M. M. Bataiev, O. Y. Sliusarenko, M. Van der Auweraer, and A. G. Vitukhnovsky, "Non-coherent exciton migration in J-aggregates of the dye THIATS: exciton-exciton annihilation and fluorescence depolarization," Chemical Physics Letters, vol. 298, no. 4-6, pp. 341-350, 1998.

[97] I. G. Scheblykin, O. Y. Sliusarenko, L. S. Lepnev, A. G. Vitukhnovsky, and M. Van der Auweraer, "Strong nonmonotonous temperature dependence of exciton migration rate in J-aggregates at temperatures from 5 to $300 \mathrm{~K}$," The Journal of Physical Chemistry: B, vol. 104, no. 47, pp. 1094910951, 2000.

[98] C. Spitz and S. Daehne, "Low temperature exciton-exciton annihilation in amphi-PIPE J-aggregates," International Journal of Photoenergy, vol. 2006, Article ID 84950, 7 pages, 2006.

[99] S. Kirstein, H. von Berlepsch, and C. Bottcher, "Photo induced reduction of noble metal ions to metal nanoparticles on tubular J-aggregates," International Journal of Photoenergy, vol. 2006, Article ID 47917, 2006.

[100] I. V. Rubtsov, K. Ebina, F. Satou, et al., "Spectral sensitization and supersensitization of AgBr nanocrystals studied by ultrafast fluorescence spectroscopy," The Journal of Physical Chemistry: A, vol. 106, no. 12, pp. 2795-2802, 2002.

[101] J.-W. Oh, S. Kumazaki, I. V. Rubtsov, T. Suzumoto, T. Tani, and K. Yoshihara, "Ultrafast energy transfer in J-aggregate on AgBr microcrystals: its dependence on dye coverage," Chemical Physics Letters, vol. 352, no. 5-6, pp. 357-362, 2002. 
[102] T. Tani, T. Suzumoto, K. Kemnitz, and K. Yoshihara, "Picosecond kinetics of light-induced electron transfer from Jaggregated cyanine dyes to silver bromide microcrystals: effect of aggregate size," The Journal of Physical Chemistry, vol. 96, no. 7, pp. 2778-2783, 1992.

[103] C. Königstein and R. Bauer, "Photoinduced hydrogen production in dilute solutions and organized assemblies," International Journal of Hydrogen Energy, vol. 18, no. 9, pp. 735741, 1993.

[104] C. Königstein, M. Neumann-Spallart, and R. Bauer, "Electrochemical investigations of a covalently bonded viologen cyanine dye-system," Electrochimica Acta, vol. 43, no. 16-17, pp. 2435-2445, 1998. 


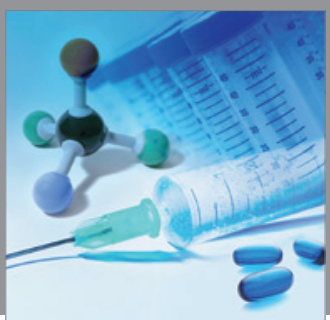

International Journal of

Medicinal Chemistry

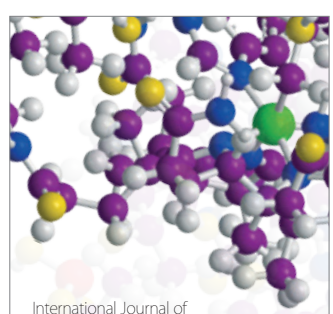

Carbohydrate Chemistry

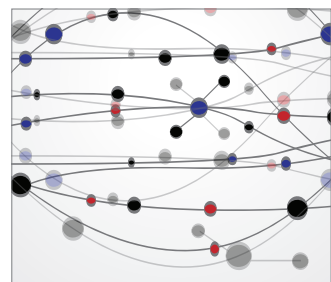

The Scientific World Journal
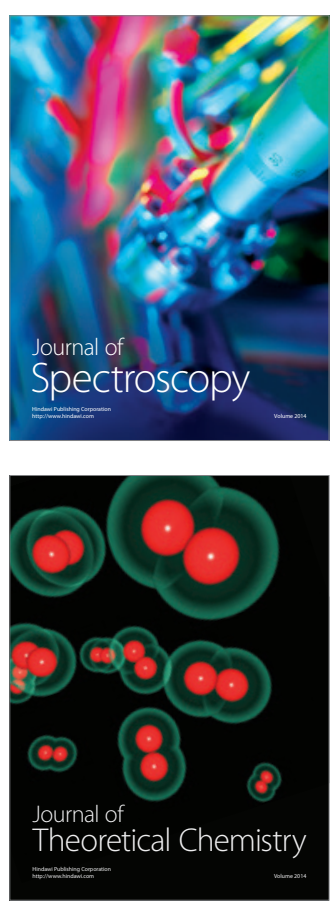
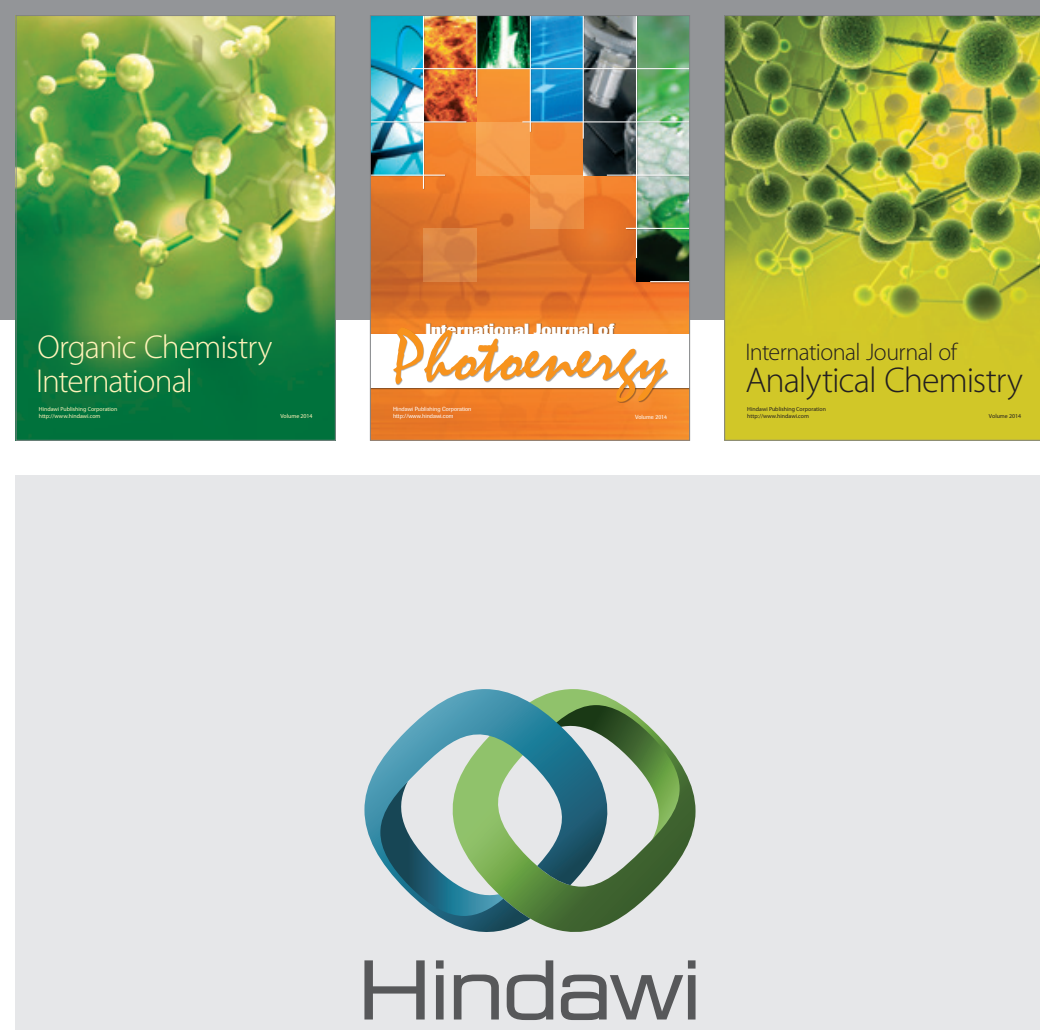

Submit your manuscripts at

http://www.hindawi.com
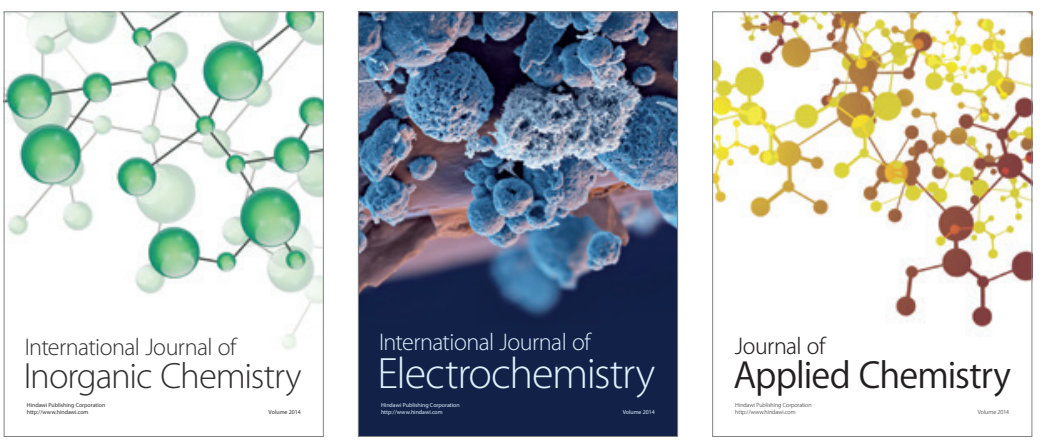

Journal of

Applied Chemistry
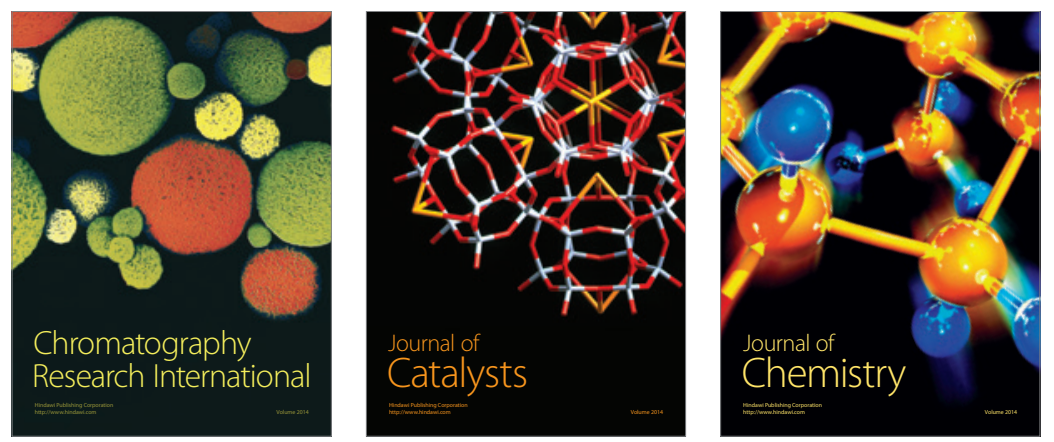
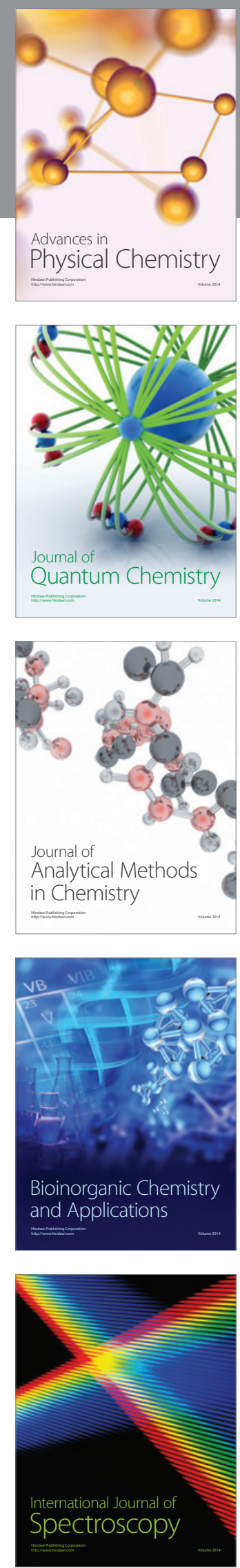